\title{
Phase diagrams of honeycomb and square nanocrystal superlattices from the nanocrystal's surface chemistry at the dispersion-air interface $\odot$
}

Cite as: J. Chem. Phys. 151, 234702 (2019); https://doi.org/10.1063/1.5128122

Submitted: 16 September 2019 . Accepted: 26 November 2019 . Published Online: 16 December 2019

Giuseppe Soligno (D), and Daniel Vanmaekelbergh

\section{COLLECTIONS}

Paper published as part of the special topic on Colloidal Quantum Dots

Note: This paper is part of the JCP Special Topic on Colloidal Quantum Dots.

This paper was selected as Featured

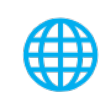

View Online
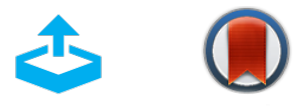

Export Citation

\section{ARTICLES YOU MAY BE INTERESTED IN}

Polymer physics across scales: Modeling the multiscale behavior of functional soft materials and biological systems

The Journal of Chemical Physics 151, 230902 (2019); https://doi.org/10.1063/1.5126852

Oxygen activation on the interface between Pt nanoparticles and mesoporous defective $\mathrm{TiO}_{2}$ during $\mathrm{CO}$ oxidation

The Journal of Chemical Physics 151, 234716 (2019); https://doi.org/10.1063/1.5131464

Effects of interface-potential smoothness and wavefunction delocalization on Auger recombination in colloidal CdSe-based core/shell quantum dots

The Journal of Chemical Physics 151, 234703 (2019); https://doi.org/10.1063/1.5125940

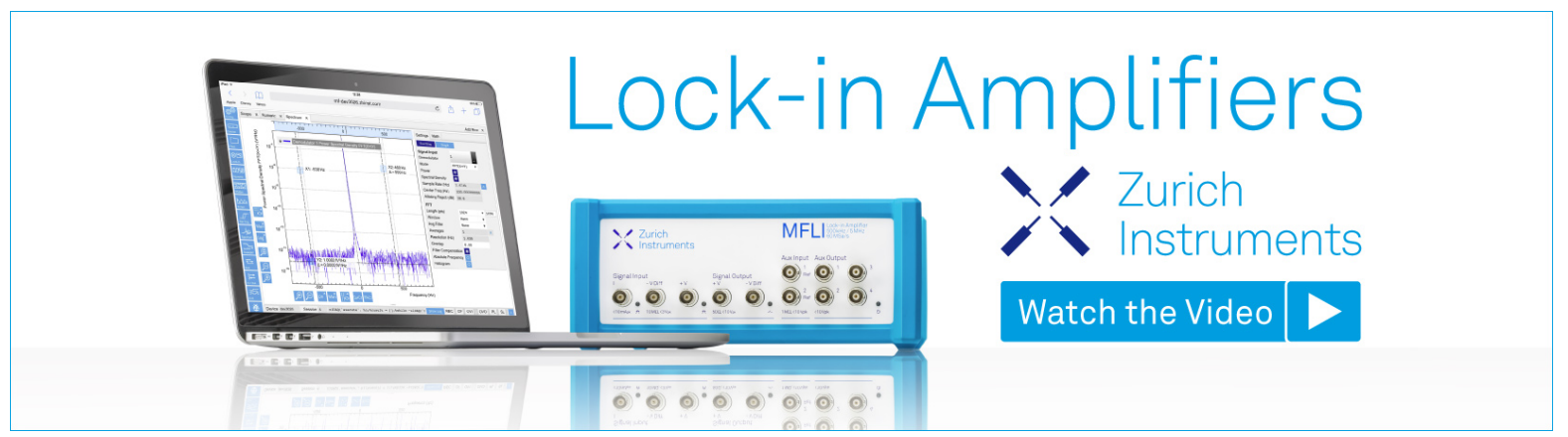




\title{
Phase diagrams of honeycomb and square nanocrystal superlattices from the nanocrystal's surface chemistry at the dispersion-air interface
}

\author{
Cite as: J. Chem. Phys. 151, 234702 (2019); doi: 10.1063/1.5128122 \\ Submitted: 16 September 2019 • Accepted: 26 November 2019 • \\ Published Online: 16 December 2019
}
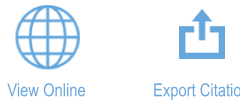

\author{
Giuseppe Soligno ${ }^{a)}$ (D) and Daniel Vanmaekelbergh
}

\begin{abstract}
AFFILIATIONS
Condensed Matter and Interfaces, Debye Institute for Nanomaterials Science, Utrecht University, Princetonplein 1 , Utrecht 3584 CC, The Netherlands
\end{abstract}

\begin{abstract}
Note: This paper is part of the JCP Special Topic on Colloidal Quantum Dots.
a)g.soligno@uu.nl
\end{abstract}

\begin{abstract}
In this work, we theoretically investigate the conditions favoring the interfacial self-assembly of PbSe nanocrystals (NCs) resulting in silicenehoneycomb superstructures. Using a coarse-grained molecular dynamics model, we study the NCs' self-assembly at the dispersion-air interface with respect to the input parameters regulating the various forces experienced by the NCs at the interface. From these results, we extrapolate detailed assembled-phase diagrams showing which ranges of the input parameters promote the formation of silicene-honeycomb superstructures and which regimes result in square geometries. Then, we use a sharp-interface numerical model to compute the energy landscape experienced by each NC at the dispersion-air interface with respect to the NC's surface chemistry. From such an energy landscape, we fit the parameters regulating the interface-adsorption forces experienced by the NCs at the interface. Combining these findings with the results presented in our assembled-phase diagrams, we find out which surface-chemistry properties of the NCs better promote the interfacial self-assembly in silicene-honeycomb superstructures, and we speculate on some experimental strategies to reach an improved control on the synthesis of PbSe silicene-honeycomb superstructures.
\end{abstract}

Published under license by AIP Publishing. https://doi.org/10.1063/1.5128122

\section{INTRODUCTION}

Low-dimensional semiconductor nanostructured materials have applications in a wide range of optoelectronic devices, such as transistors, light-emitting diodes, photovoltaic cells, lasers, thermoelectric modules, and sensors. ${ }^{1,2}$ Interfacial self-assembly of semiconductor nanocrystals (NCs) has emerged in the last decade as a promising bottom-up route to build these materials.

Recently, a semiconductor with a silicene-honeycomb nanogeometry has been synthesized by the interfacial self-assembly of PbSe NCs. ${ }^{31}$ The NCs have a size of $5-6 \mathrm{~nm}\left(\sim 10^{4}\right.$ atoms $)$, a rocksalt crystal structure, and a truncated-cube shape with $\{100\},\{110\}$, and $\{111\}$ facets. ${ }^{32}$ Such NCs form an ordered monolayer at the dispersion-air interface and, eventually, bind to each other by the formation of a crystalline neck between opposite $\{100\}$ facets, a process known as oriented attachment. ${ }^{23,33,34}$ In the final superstructure, the NCs are epitaxially connected and with aligned atomic lattices, forming effectively a single crystal with both a short-range periodicity, due to the atomic lattice, and a long-range silicenehoneycomb periodicity of the NCs' position. Semiconductors with the honeycomb nanogeometry are of particular interest, since calculations predict for them Dirac-type electronic conduction and valence bands, with the semiconductor bandgap preserved. ${ }^{3}$ Therefore, such materials would combine the properties of semiconductors with those of graphene, offering novel opportunities for a wide range of optoelectronic applications. Such a "bottomup" route for producing nanogeometric-honeycomb semiconductors is complementary to the more expensive lithographic patterning methods, as self-assembly allows for smaller periodicity and, thus, stronger electronic bonds. However, despite recent improvements, 


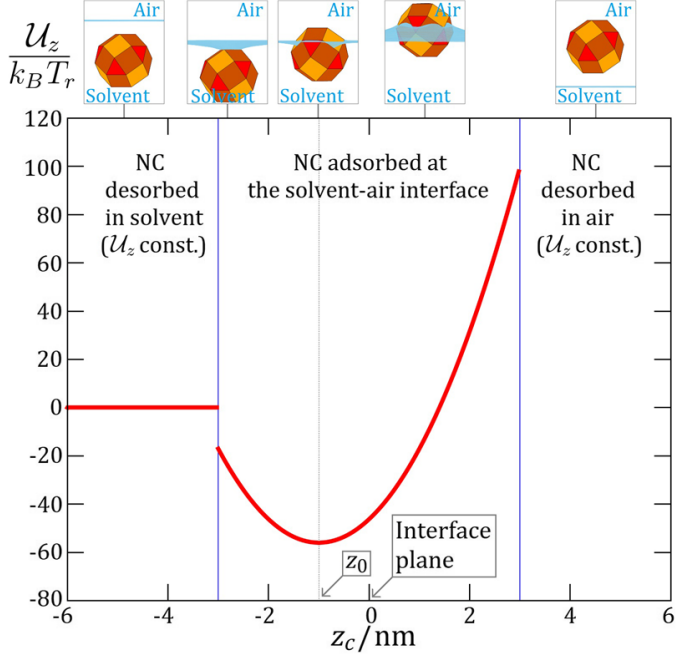

FIG. 1. Illustrative plot of the external potential $\mathcal{U}_{z}\left(z_{c}\right)$ [Eq. (1)] applied, in our coarse-grained molecular dynamics model, to each $\mathrm{NC}$ close to the interface plane $z=0$, to reproduce the interface-adsorption forces binding the NCs at the interface. $z_{c}$ is the $z$ coordinate of the NC's center of mass, i.e., \pm the distance of the NC from the interface plane. The 3D sketches on the top of the plot of $\mathcal{U}_{z}\left(z_{c}\right)$ help in visualizing the NC's position at the interface with respect to $z_{c} . \mathcal{U}_{z}\left(z_{c}\right)$ is plotted in units of the thermal energy at room temperature, setting $u_{z}=0.4 \times 10^{-19} \mathrm{~J}$, $z_{d}=3 \mathrm{~nm}$, and $z_{0}=-1 \mathrm{~nm}$, which are within the expected experimental range (see our predictions for an interface-adsorbed NC in Sec. III). The constants $E_{s}$, $E_{1}$, and $E_{a}$ of $\mathrm{Eq}$. (1) (which do not affect the molecular dynamics simulations of Sec. II) in this plot are $E_{s}=0, E_{a}=20.0 \times 10^{-19} \mathrm{~J}$, and $E_{1}=-2.3 \times 10^{-19} \mathrm{~J}$, i.e., in line with the expected values of the interface-adsorption energy $E$ [see Eq. (11) in Sec. III] of a NC desorbed in solvent, desorbed in air, and at equilibrium at the interface, respectively. the PbSe silicene-honeycomb superlattices currently synthesized are still afflicted by a high degree of structural disorder and poor reproducibility, ${ }^{38}$ and many open questions on the formation process still remain.

In recent work, ${ }^{40}$ we presented a coarse-grained molecular dynamics model that illustrates the basic mechanism of formation of silicene-honeycomb superstructures by the self-assembly of interface-adsorbed PbSe NCs. This model successfully reproduces the self-assembly of NCs in silicene-honeycomb superstructures. The key ingredients of the model are the following (for details, see Ref. 40):

(i) PbSe NCs are modeled by a polybead structure reproducing a rhombicuboctahedron of size $6 \mathrm{~nm}$ (see Figs. 1-3), that is, the typical shape of rocksalt PbSe NCs.

(ii) The solvent is treated implicitly by modeling the NCs' Brownian motion.

(iii) The NCs' dynamics is simulated using bead-bead pair potentials to reproduce NC-NC short-range facet-specific attractive and repulsive interactions.

(iv) External potentials are applied to the NCs' beads to mimic the interface-adsorption forces experienced by NCs at a fluid-fluid interface.

By opportunely tuning the bead-bead interactions [point "(iii)"], the NCs are allowed to attract and attach to each other by opposite $\{100\}$ facets only, i.e., the light-orange-colored facets in the NC's sketches in Figs. 1-3. After the NCs' synthesis, the NCs are dispersed in a solvent (e.g., toluene) and ligand molecules (typically, oleic acid) are chemisorbed at the NCs' surface and keep the NC cores far apart from each other. On the NCs' $\{100\}$ facets, the ligand

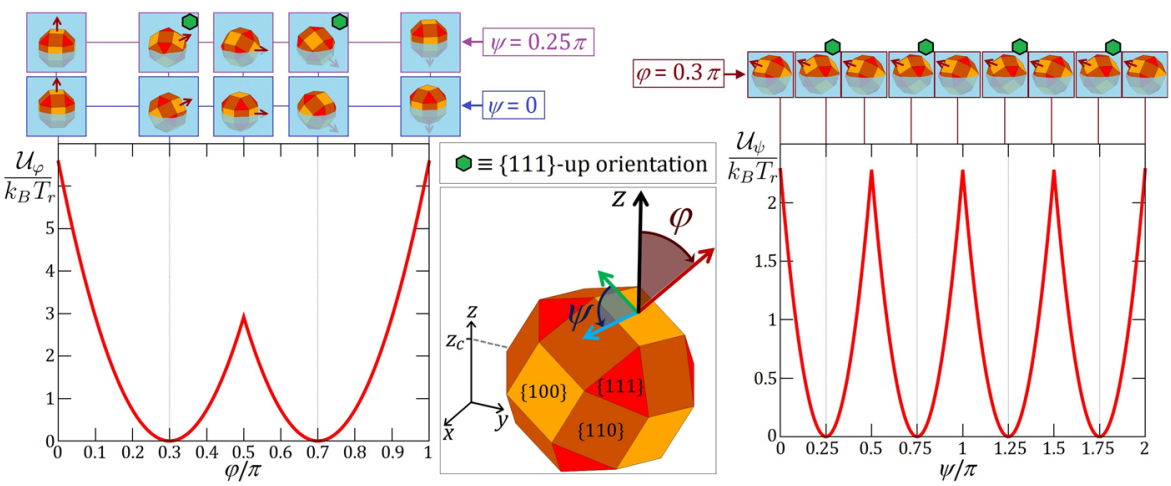

FIG. 2. Illustrative plots of the interface-adsorption potentials $\mathcal{U}_{\varphi}(\varphi)$ [Eq. (2)] and $\mathcal{U}_{\psi}(\psi)$ [Eq. (3)] applied, in our coarse-grained molecular dynamics model, ${ }^{40}$ to each NC close to the interface plane $z=0$, to reproduce the interface-adsorption forces orienting the NCs with a $\{111\}$ upward at the interface. The inset in the center shows a rhombicuboctahedron NC, with its $\{100\}$ facets colored in light orange, its $\{110\}$ facets colored in dark orange, and its $\{111\}$ facets colored in red. $\varphi \in[0, \pi]$ is the polar angle of the NC's vertical axis (corresponding to one of the NC' $\langle 100\rangle$ directions and indicated in the sketches by a red arrow). $\psi \in[0,2 \pi)$ is the NC's internal Euler angle around its vertical axis. $z_{c}$ is the $z$ coordinate of the NC's center of mass, i.e., \pm the distance of the NC from the interface plane. The 3D sketches on the top of the plots of $\mathcal{U}_{\varphi}(\varphi)$ and $\mathcal{U}_{\psi}(\psi)$ show the NC's orientation at the interface with respect to $\varphi$ (for a given value of $\psi$ ) and $\psi$ (for a given value of $\varphi$ ), respectively. The 3D sketches marked by a green hexagon on their top-right corner correspond to the \{111\}-up orientation of the NC at the interface (see also Fig. 3 ), that is, the minimum- $\mathcal{U}_{\varphi}$, minimum- $\mathcal{U}_{\psi}$ orientation. This is obtained by setting in Eq. (2): $\varphi_{0}=0.3 \pi$ and $\varphi_{0}=0.7 \pi$, and in Eq. (3): $\psi_{0}=0.25 \pi, \psi_{0}=0.75 \pi, \psi_{0}=1.25 \pi$, and $\psi_{0}=1.75 \pi$ (during a simulation with our coarse-grained molecular dynamics model, to calculate $\mathcal{U}_{\varphi}$ and $\mathcal{U}_{\psi}$, we select the closest values of $\varphi_{0}$ and $\psi_{0}$ to the NC's $\varphi$ and $\psi$ at the current time step). $\mathcal{U}_{\varphi}(\varphi)$ and $\mathcal{U}_{\psi}(\psi)$ are plotted in units of the thermal energy at room temperature, setting $u_{\varphi}=3.0 \times 10^{-19} \mathrm{~J}$ and $u_{\psi}=1.5 \times 10^{-19} \mathrm{~J}$, which are within the expected experimental range (see our predictions for an interface-adsorbed NC in Sec. III B), and $z_{c}=z_{0}$. 

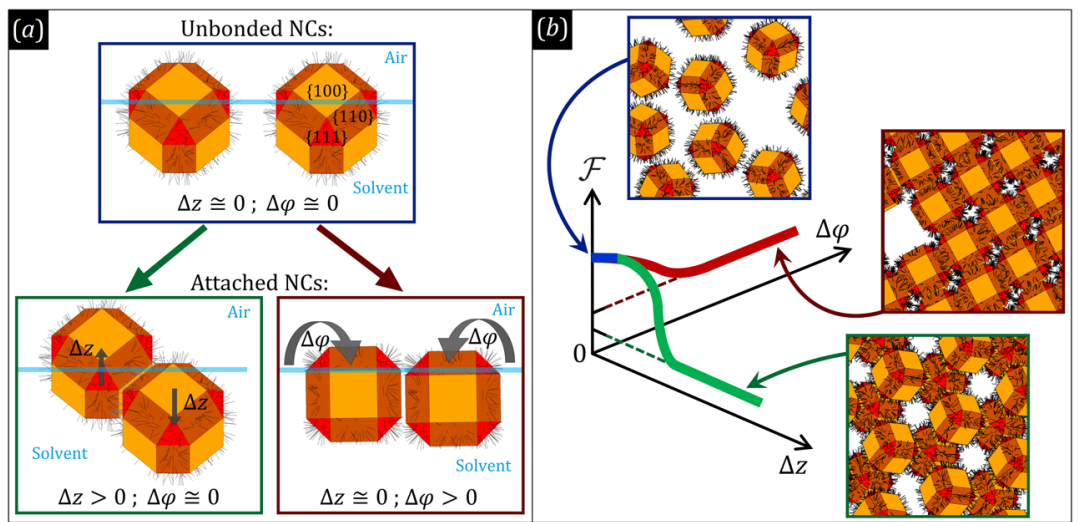

FIG. 3. (a) Sketch of the self-assembly mechanism of interface-adsorbed NCs in silicene-honeycomb or square superstructures. An isolated NC at the interface is, at equilibrium, oriented with a $\{111\}$ facet (red) upward and with center of mass at a height $z_{c}$ on the interface plane $z=0$ [see the top panel in (a)]. The NCs can decrease their free energy $\mathcal{F}[\mathrm{Eq}$. (4)] by assembling in either silicene-honeycomb superstructures, by shifting their height at the interface plane by an average distance $\Delta z$, half of the NCs moving upward and half of the NCs downward [see bottom-left panel in (a)], or in square superstructures, by changing orientation $\varphi$ at the interface by an average angle $\Delta \varphi$, shifting from \{111\}-up to \{100\}-up, i.e., with a light orange facet upward [see the bottom-right panel in (a)]. (b) These self-assembly mechanisms are possible only if the energy penalties paid in $\Delta \varphi$ or $\Delta z$ are compensated by a higher energy gain from the NC-NC attachment. The NCs follow the path leading to the lowest total free energy $\mathcal{F}$ [Eq. (4)]. The insets in (b) are sketches of the interface top view for NCs not bound $(\Delta \varphi \simeq 0, \Delta z \simeq 0)$, NCs assembled in silicene-honeycomb superstructures $(\Delta \varphi \simeq 0, \Delta z>0)$, and $\mathrm{NCs}$ assembled in square superstructures $(\Delta \varphi>0, \Delta z \simeq 0)$.

chemisorption is weaker, resulting in ligand desorption from these facets under opportune experimental conditions, due to the presence of ethylene glycol. ${ }^{30,38}$ In such a way, attractive chemical interactions between opposed $\{100\}$ facets become possible. The bond energy $\mathcal{E}$ between two NCs attached by opposite $\{100\}$ facets is an input parameter of our coarse-grained molecular dynamics model. The attractive chemical interactions between the (nearly) ligand-free $\{100\}$ facets of the NCs are the driving force of the self-assembly. However, for NCs dispersed in the solvent, such a self-assembly would lead to a simple cubic superstructure. The occurrence of twodimensional superstructures indicates that the NCs assemble and interact with each other while they are confined at an interface. In line with this, one of us calculated that, for NCs at the NCs' solvent (toluene)-air interface, the $\mathrm{NC}$-interface bonding energy is at least $\sim 10 k_{B} T_{r}$ or higher, ${ }^{40,41}$ with $k_{B}$ the Boltzmann constant and $T_{r}$ room temperature. Recent combined experiment-theory results ${ }^{41}$ indicate that the NCs do not adsorb at the liquid substrate (ethylene glycol)-solvent interface nor at the liquid substrate (ethylene glycol)air interface upon evaporation of the solvent. Thus, we assume that the NCs' assembly and oriented attachment, leading to the PbSe silicene-honeycomb superstructures, occur at the solvent-air interface. Such an interface is represented in our coarse-grained molecular dynamics model by external potentials applied to the NCs [point "(iv)"]. First, the interface-adsorbed NCs are forced to stay at the solvent-air interface plane. Introducing a Cartesian coordinate system $x, y, z$, with $z=0$ representing the solvent-air interface plane, the $z$ coordinate of the center of the mass position of each $\mathrm{NC}$, say $z_{c}$, is bonded to the solvent-air interface by the harmonic potential,

$$
\mathcal{U}_{z}\left(z_{c}\right) \equiv\left\{\begin{array}{l}
E_{s}, \text { if } z_{c}<-z_{d}, \\
E_{1}+u_{z}\left(\frac{z_{c}-z_{0}}{\mathrm{~nm}}\right)^{2}, \text { if }\left|z_{c}\right| \leq z_{d}, \\
E_{a}, \text { if } z_{c}>z_{d},
\end{array}\right.
$$

where $u_{z}$ is an input parameter, $z_{0}$ is the equilibrium height of each $\mathrm{NC}$ 's center of mass at the interface plane, $z_{d}$ is the largest distance of the NC's center of mass from the interface plane at which a NC is considered adsorbed at the interface, and $E_{s}, E_{1}$, and $E_{a}$ are constants. A plot of $\mathcal{U}_{z}$, using typical experimental values, is shown in Fig. 1. Second, the interface-adsorbed NCs are forced to stay oriented with a $\{111\}$ facet (red-colored facets in the NC's sketches in Figs. 1-3) pointing upward at the interface, i.e., approximately parallel to the interface plane. We define (see Fig. 2) $\varphi \in[0, \pi]$ as the polar angle of a NC's vertical axis (given by one of its $\langle 100\rangle$ directions) with respect to the interface plane and $\psi \in[0,2 \pi)$ as the NC's internal Euler angle around its vertical axis. Each interface-adsorbed NC experiences the potentials

$$
\begin{aligned}
& \mathcal{U}_{\varphi}(\varphi)=\mathcal{u}_{\varphi}\left[\varphi-\varphi_{0}(\varphi)\right]^{2} \xi\left(z_{c}\right), \\
& \mathcal{U}_{\psi}(\psi)=u_{\psi}\left[\psi-\psi_{0}(\psi)\right]^{2} \xi\left(z_{c}\right),
\end{aligned}
$$

where $u_{\varphi}$ and $u_{\psi}$ are input parameters, and $\varphi_{0}$ and $\psi_{0}$ are the equilibrium values of $\varphi$ and $\psi$, respectively, i.e., corresponding to the $\{111\}$-up orientation of the NCs at the interface. Plots of $\mathcal{U}_{\varphi}$ and $\mathcal{U}_{\psi}$, using typical experimental values, are shown in Fig. 2 . The function $\xi\left(z_{c}\right)$ in Eqs. (2) and (3) is 1 for $z_{c}=z_{0}, 0$ for $\left|z_{c}\right|>z_{d}$, a linear interpolation of these values for $\left|z_{c}\right|<z_{d}$, and is used to switch on (or off) the orientation forces when a NC adsorbs to (or desorbs from) the interface. The interface-adsorption potentials $\mathcal{U}_{z}\left(z_{c}\right), \mathcal{U}_{\varphi}(\varphi)$, and $\mathcal{U}_{\psi}(\psi)$ applied in our model to interfaceadsorbed NCs are meant to approximately represent the forces experienced by NCs at a fluid-fluid interface and are estimated in Ref. 40 for PbSe NCs covered by oleic acid ligands at the tolueneair interface and in this work; see Sec. III, for various NC's surface chemistry. We remark that our coarse-grained molecular dynamics model ${ }^{40}$ can be applied to study the NCs' self-assembly at any fluid-fluid interface, as long as the NCs' adsorption properties at this 
interface are known [i.e., the input parameters of our coarse-grained molecular dynamics model regulating $\mathcal{U}_{\varphi}(\varphi), \mathcal{U}_{\psi}(\psi)$, and $\mathcal{U}_{z}\left(z_{c}\right)$, which can be predicted, e.g., by the numerical model illustrated in Sec. III].

While the NCs keep the $\{111\}$-up orientation at the interface, because of the potentials $\mathcal{U}_{\varphi}$ and $\mathcal{U}_{\psi}$ [Eqs. (2) and (3)], and stay inplane, because of the potential $\mathcal{U}_{z}$ [Eq. (1)], they cannot attach by opposite $\{100\}$ facets for clear geometrical reasons [see Fig. 3(a)]. The NC-NC attachment by other NCs' facets would be geometrically possible in this situation, but is prevented by the ligand molecules chemisorbed on these facets (represented in our model by bead-bead soft repulsive pair potentials; see Ref. 40). To make NC-NC attachment by opposite $\{100\}$ facets possible while the NCs keep the $\{111\}$-up orientation at the interface, half of the NCs have to move slightly below their equilibrium height at the interface plane and half of the NCs slightly above [see Fig. 3(a)]. This procedure can occur only if the NCs' energy gain in attaching by opposite $\{100\}$ facets ( $\sim \mathcal{E}$ per NC-NC bond) is larger than the NCs' energy penalty in $\mathcal{U}_{z}$ due to the NCs' shift from their equilibrium height and only if the energy barriers encountered are comparable with the thermal energy. If these conditions occur, silicene-honeycomb superstructures form at the interface (see Ref. 40). Alternatively, the interfaceadsorbed NCs can attach by opposite $\{100\}$ facets while remaining in-plane (i.e., without an energy penalty in $\mathcal{U}_{z}$ ) by shifting from their equilibrium $\{111\}$-up orientation and orienting with a $\{100\}$ facet upward at the interface [see Fig. 3(a)]. This procedure can occur only if the NCs' attachment energy gain $(\sim \mathcal{E}$ per NC-NC bond) is larger than the NCs' energy penalty in $\mathcal{U}_{\varphi}$ and $\mathcal{U}_{\psi}$ due to the NCs' shift from their equilibrium orientation and only if the energy barriers encountered are comparable with the thermal energy. If these conditions occur, square superstructures form at the interface (see Ref. 40). If neither of these two self-assembly mechanisms is energetically favorable, the interface-adsorbed NCs retain their $\{111\}$-up orientation while remaining in-plane; therefore, they cannot form $\{100\}$ $\{100\}$ bonds and no oriented attachment occurs (but dense ordered monolayers of not-attached NCs can be found at high packing fractions, with the ordering driven, in this case, mainly by the NCs' entropy $^{42,43}$ ).

To sum up our coarse-grained molecular dynamics model, ${ }^{4}$ the NCs are adsorbed at a fluid-fluid interface, and this interface forces each NC to orient with a $\{111\}$ facet upward and to stay inplane with the other NCs. Only the NCs' $\{100\}$ facets attract each other, while the remaining NCs' facets, i.e., $\{110\}$ and $\{111\}$, do not attract each other (since, at some point in the experiments, ligand molecules are expected to be chemisorbed only on the NCs' $\{110\}$ and $\{111\}$ facets). So, the interface-adsorbed NCs can gain energy by attaching by opposite $\{100\}$ facets $(\sim \mathcal{E}$ per NC-NC bond). However, to attach by opposite $\{100\}$ facets [see Fig. 3(a)] the NCs need to pay an energy penalty either to rotate away from their equilibrium orientation [i.e., in the potential $\mathcal{U}_{\varphi}+\mathcal{U}_{\psi}$; see Eqs. (2) and (3)] or to move away from their equilibrium height at the interface plane and lose their in-plane geometrical configuration [i.e., in the potential $\mathcal{U}_{z}$; see Eq. (1)]. Consequently, three scenarios can occur: self-assembly in silicene-honeycomb superstructures, self-assembly in square superstructures, and no self-assembly. Such situations have all been experimentally found. ${ }^{26,30,31,38}$ The scenario that occurs in the model depends on the interplay between the parameters regulating the various forces:
- $\mathcal{E}$, which regulates the strength of the attractive forces between opposite $\{100\}$ facets of nearby NCs,

- $u_{z}$, which regulates the strength of the forces due to $\mathcal{U}_{z}$ [Eq. (1)] that keeps the interface-adsorbed NCs in-plane,

- $u_{\varphi}, u_{\psi}$, which regulate the strength of the forces due to $\mathcal{U}_{\varphi}$ and $\mathcal{U}_{\psi}$ [Eqs. (2) and (3)] that keep the interface-adsorbed NCs in the $\{111\}$-up orientation, and

- temperature $T$ (introduced in the model by the NCs' Brownian motion), which sets the thermal energy scale.

This self-assembly mechanism, introduced in detail in Ref. 40, is synthetically sketched in Fig. 3(b). The total free energy $\mathcal{F}$ of $N$ $\mathrm{NCs}$ at the solvent-air interface, within a fixed volume $V$, is

$$
\mathcal{F}=\mathcal{U}_{\text {tot }}-T \cdot \mathcal{S}_{N}
$$

where $T$ is the temperature, $\mathcal{S}_{N}$ is the entropy of the $N$ NCs, and $\mathcal{U}_{\text {tot }} \equiv \mathcal{U}_{\text {kin }}+\mathcal{U}_{\text {pot }}$ is the total internal energy of the system, with $\mathcal{U}_{\text {kin }}$ the NCs' total kinetic energy and

$$
\mathcal{U}_{\text {pot }} \equiv \mathcal{U}_{z}+\mathcal{U}_{\varphi}+\mathcal{U}_{\psi}+\mathcal{U}_{N C-N C},
$$

the total potential energy of the NCs, where $\mathcal{U}_{z}, \mathcal{U}_{\varphi}$, and $\mathcal{U}_{\psi}$ are (implicitly) summed over all NCs in Eq. (5), and $\mathcal{U}_{N C-N C}$ is the total potential due to the NC-NC interactions. So,

$$
\mathcal{U}_{\mathrm{NC}-\mathrm{NC}} \simeq M \mathcal{E}
$$

with $M$ the total number of bonds formed by NCs attached by two opposite $\{100\}$ facets. The NCs evolve toward configurations with lower $\mathcal{F}$, which can be achieved by bonding by opposite $\{100\}$ facets ( $\mathcal{F}$ decreases by $\sim \mathcal{E}$ for each new NC-NC bond formed). However, to form $\{100\}-\{100\}$ bonds, the NCs have to pay an energy penalty, either in $\mathcal{U}_{z}$, to change their height at the interface plane, which leads to silicene-honeycomb superstructures, or in $\mathcal{U}_{\varphi}+\mathcal{U}_{\psi}$, to change their orientation from $\{111\}$-up to $\{100\}$-up, which leads to square superstructures. The energy penalty in $\mathcal{U}_{z}$, for shifting the height of a NC at the interface of $\sim 1$ to $2 \mathrm{~nm}$, i.e., enough to feel the attractive forces of the $\{100\}$ facets of nearby NCs, is $\sim 5$ to $10 k_{B} T_{r}$ for typical experimental parameters (see Fig. 1). The energy penalty in $\mathcal{U}_{\varphi}+\mathcal{U}_{\psi}$, for shifting the orientation of an interfaceadsorbed NC from $\{111\}$-up to $\{100\}$-up, is $\sim 3 k_{B} T_{r}$ for typical experimental parameters (see Fig. 2). These energy penalties determine which path, either to silicene-honeycomb or to square superstructures, is energetically more favorable for the interface-adsorbed NCs or whether the interface-adsorbed NCs do not attach by $\{100\}$ $\{100\}$ bonds at all (if the energy gain, $\simeq \mathcal{E}$ for each NC-NC bond formed, is too low compared to the energy penalties to be paid in $\mathcal{U}_{\varphi}+\mathcal{U}_{\psi}$ or in $\left.\mathcal{U}_{z}\right)$. Note that, in our simulations, NC-NC bonds are irreversible (see Sec. II). However, few-NC aggregates can shift from silicene-honeycomb to square precursors or vice versa (by changing the few-NC aggregate orientation at the interface), allowing the NCs to explore the energy landscape and self-assemble in the superstructure, either square or silicene-honeycomb, with the lowest total free energy $\mathcal{F}$.

In this work, we aim at investigating how the experimental conditions can be tuned to favor the formation of silicene-honeycomb superstructures. The parameter $\mathcal{E}$ is the most difficult to control in the experiments. $\mathcal{E}$ could be regulated, e.g., by changing the NC's size 
or compound, but this would then probably require to fully recalibrate most of the many other experimental parameters involved in the synthesis procedure: solvent, ligands, evaporation time, etc. The parameters $u_{z}, u_{\varphi}$, and $u_{\psi}$ seem to be the more convenient first choice for our investigation, since they can be regulated experimentally by slightly tuning either the surface tensions in the system or the shape of the NCs, which should not drastically affect the remaining experimental conditions.

- First, in Sec. II, the coarse-grained molecular dynamics model presented in Ref. 40 (and briefly reviewed in the Introduction) is used to quantitatively investigate which range of values for the model's input parameters $\mathcal{E}, u_{z}, u_{\varphi}$, and $u_{\psi}$, leads to the silicene-honeycomb self-assembly or to square self-assembly, exploring the parameter space more extensively than in Ref. 40.

- Second, in Sec. III, a sharp-interface macroscopic model is briefly illustrated (Sec. III A) and then used to numerically predict the interface-adsorption energetic potentials experienced by an isolated NC at a flat fluid-fluid interface with respect to the NC's shape and surface chemistry (Sec. III B). In the cases where the $\{111\}$-up orientation is stable for the single-adsorbed NC, the parameters ${u_{z}}_{z} \mathcal{u}_{\varphi}$, and $u_{\psi}$ are fitted from the predicted interface-adsorption energy landscape, to find out, on the basis of the results of Sec. II, which combinations of NC's surface chemistry and shape are better to induce self-assembly in silicene-honeycomb superstructures or in square superstructures.

- Finally, in Sec. IV, we discuss our theoretical predictions and, on the basis of these, speculate some experimental guidelines to optimize the synthesis of silicene-honeycomb superstructures.

\section{SELF-ASSEMBLY RESULTS}

In this section, we use the coarse-grained molecular dynamics model introduced in Ref. 40 to simulate the self-assembly of PbSe NCs of $6 \mathrm{~nm}$ size at the dispersion-air interface, with respect to the parameter $\mathcal{E}$, regulating the attractive forces between the $\{100\}$ facets of the NCs, and the parameters $u_{z}, u_{\varphi}$, and $u_{\psi}$, regulating the interface-adsorption forces orienting the interface-adsorbed NCs with a $\{111\}$ facet upward and keeping them in-plane (see Sec. I and Ref. 40 for details).

We consider 7 different values of $\mathcal{E}$ (bond energy for two NCs attached by opposite $\{100\}$ facets), ranging from $-0.7 \times 10^{-19} \mathrm{~J}$ to $-1.3 \times 10^{-19} \mathrm{~J}$ (i.e., from $\simeq-17 k_{B} T_{r}$ to $\simeq-32 k_{B} T_{r}$ ), that is, the expected order of magnitude for $\sim 6$-nm-sized NCs bonded by oriented attachment. ${ }^{44}$ Therefore, formed NC-NC bonds are irreversible, since all our simulations are conducted at room temperature. For each value of $\mathcal{E}$, we consider 56 values of $u_{\varphi}$, ranging from $0.5 \times 10^{-19} \mathrm{~J}$ to $6.0 \times 10^{-19} \mathrm{~J}$, and 16 values of $u_{z}$, ranging from $0.05 \times 10^{-19} \mathrm{~J}$ to $0.80 \times 10^{-19} \mathrm{~J}$. The experimental values are expected to fall within these ranges; see Sec. III. So, in total, we perform 6272 simulations. In each simulation, we consider 16 NCs initially disposed in a dilute hexagonal monolayer at the interface, and we simulate their dynamics for $0.1 \mu \mathrm{s}$ (that is, enough time for $16 \mathrm{NCs}$ to form a stable aggregate, if they can attach to each other) at room temperature. The value of $u_{\psi}$ is always set to $u_{\psi}=u_{\varphi} / 2$, that is, approximately, the typical experimental situation; see Sec. III. In Ref. 40, we used the condition $u_{\psi}=u_{\varphi}$ (instead of $u_{\psi}=u_{\varphi} / 2$ ) in our coarse-grained molecular dynamics simulations, but we believe that the difference between these two choices has a minimal effect on the NCs' behavior at the interface. Periodic boundary conditions are applied in the $x$ and $y$ directions, i.e., parallel to the interface plane $z=0$, with period $32 \mathrm{~nm}$. The remaining parameters of our coarsegrained molecular dynamics model are set as in Ref. 40, except for the NC-NC pair capillary interaction potential, which is here deactivated, since in Ref. 40, we show it is negligible (see Ref. 40 for the model's details).

To quantify the final assembled phase of the NCs in each simulation, we define two order parameters, $e \in[0,1]$, to measure the degree of oriented attachment performed by the NCs $(e=0$ meaning no self-assembly at all, $e=1$ meaning many NC-NC bonds are formed), and $s \in[0,1]$ to measure the kind of self-assembled phase formed by the NCs ( $s=0$ meaning pure silicene-honeycomb superstructures are formed, $s=1$ meaning pure square superstructures are formed). The parameter $e$ is defined as

$$
e \equiv \min \left\{\frac{\left|\mathcal{U}_{L J}\right|}{N \cdot \mathcal{E}}, 1\right\}
$$

where " $\min \{x, y\}$ " means the minimum between $x$ and $y, \mathcal{U}_{L J}$ is the total potential inducing the NC-NC attractive interactions, i.e., summed over all the bead-bead attractive interactions (see Ref. 40 for details), and $N$ is the total number of NCs (here, $N=16$ ). So, basically, $e=0$ means that not even one NC-NC bond has been formed. The more the NCs bond together, the more $e$ increases, up to a maximum of $e=1$ corresponding to $\sim N$ NC-NC bonds (which is roughly the maximum number of bonds that can be reached by 16 aggregated NCs). The parameter $s$ is defined as

$$
s \equiv \frac{1}{N} \sum_{i=1}^{N} \min \left\{\frac{\varphi_{i}-\varphi_{0}(i)}{0.2 \pi}, 1\right\},
$$

where $\varphi_{i}$ is the polar angle $\varphi$ of the $i$ th NC and $\varphi_{0}(i)$ is the minimum$\mathcal{U}_{\varphi}$ value of $\varphi_{i}$ closest to $\varphi_{i}$ (see Fig. 2). So, $s=0$ when all NCs are in the $\{111\}$-up orientation, that is, the condition to form silicenehoneycomb superstructures, and $s$ is close to 1 when all NCs are far from the $\{111\}$-up orientation, i.e., in the $\{100\}$-up orientation, that is, the condition to form square superstructures. To recognize the final assembled phase of the NCs, both order parameters $e$ and $s$ need to be considered, since three outcomes for the NCs' selfassembly are possible: square superstructures, silicene-honeycomb superstructures, and no self-assembly. Only one of these two order parameters is not enough to univocally determine the NCs' phase. In addition, in many of the combinations of $\mathcal{E}, u_{z}, u_{\varphi}$, and $u_{\psi}$ considered, the NCs self-assemble in a combination of these three possible phases.

In Fig. 4, we sum up our new results in an assembled-phase diagram, plotting the color-coded values of $e$ and $s$, for each simulation performed, with respect to $\mathcal{E}, u_{z}$, and $u_{\varphi}$ (and the numerical values of $e$ and $s$ obtained in each simulation are reported in the supplementary material; see Tables S1-S21). The trend reported in Ref. 40 is confirmed here in much more detail: silicene-honeycomb superstructures (corresponding to $e$ close to 1 and $s$ close to 0 ) are favored by a high $u_{\varphi}$ and a low $u_{z}$, with $\mathcal{E}$ setting the thresholds of $u_{\varphi}$ and 


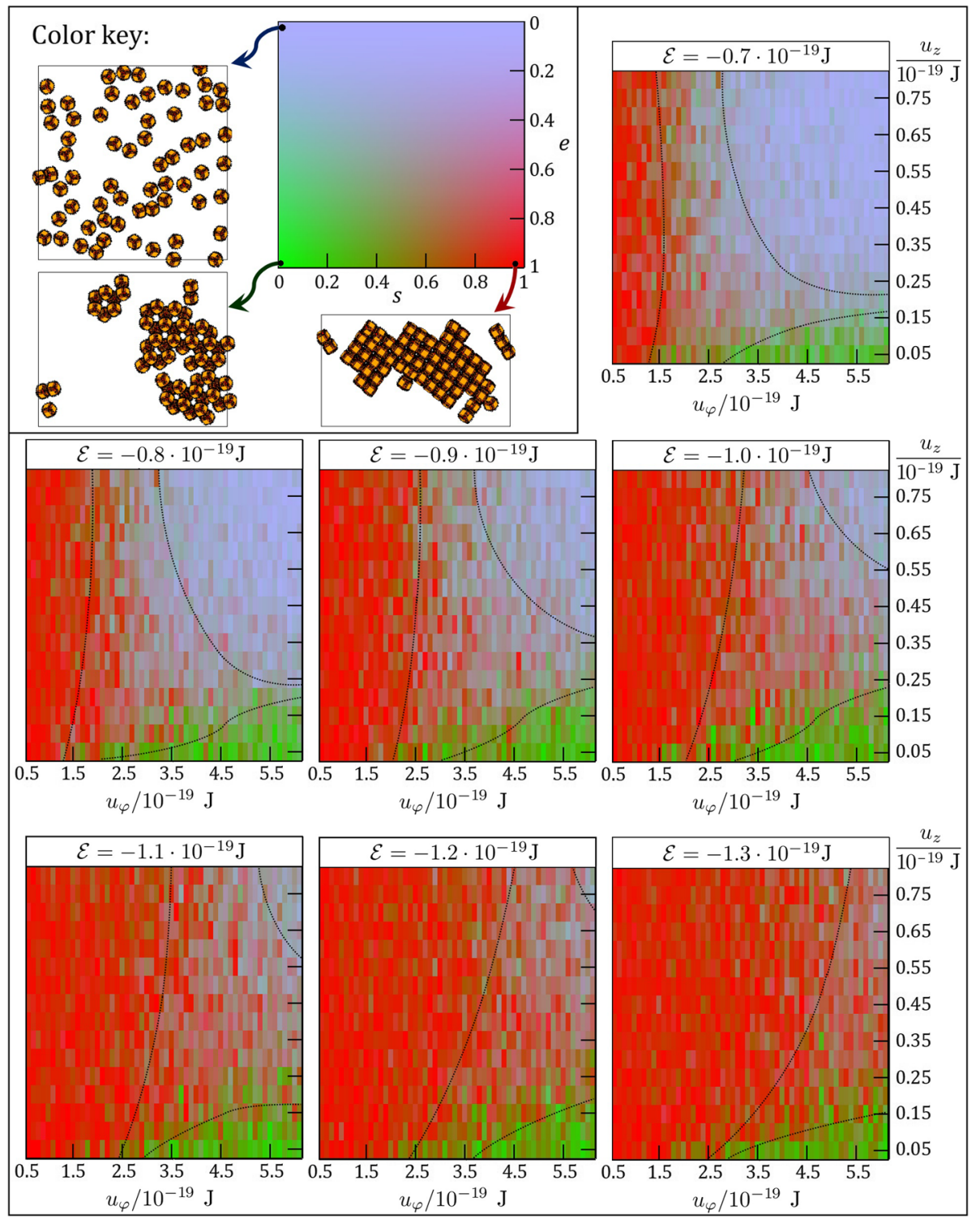

FIG. 4. Assembled-phase diagrams showing the results with our coarsegrained molecular dynamics model for the self-assembly of 6-nm-sized PbSe NCs at the dispersion-air interface at room temperature, with respect to the model input parameters $u_{\varphi}, u_{z}$, and $\mathcal{E}$ (see Sec. I). Each small box in the diagrams corresponds to a $0.1 \mu \mathrm{s}$-long simulation of 16 NCs initially dispersed at the interface. In total, 6272 of such simulations have been performed to draw these diagrams. The box color indicates the values of the order parameters $e[$ [Eq. (7)] and $s[\mathrm{Eq}$. (8)] for the obtained NCs' configuration; see the color key in the top-left inset (the NCs sketches are only illustrative). The numerical values of $e$ and $s$ for each box are reported in the supplementary material; see Tables S1-S21. If $e$ is close to 0 (blue color), the NCs did not bind. If $e$ is close to 1 , the NCs did assemble in either square superstructures (red color, corresponding to $s$ close to 1 ) or silicene-honeycomb superstructures (green color, corresponding to $s$ close to 0 ). As a guide for the eye, we added by hand arbitrary dotted lines to highlight pure silicenehoneycomb areas (majorly green), pure square superstructure areas (majorly red), pure no-assembly areas (majorly blue), and mixed-phase areas (mixed colors). In the supplementary material (see Fig. S27), we show a snapshot of the final NCs' configuration for some of the simulations performed.

$u_{z}$ above and below which, respectively, the silicene-honeycomb is formed. In Sec. III, we predict how $u_{\varphi}$ and $u_{z}$ are affected by the surface tensions in the system, to investigate which combination of surface tensions leads closer to the regime where silicene-honeycomb superstructures are favored, i.e., the areas with more green in Fig. 4. In the supplementary material, we show, for illustrative purpose, a snapshot of the final NCs' configuration obtained in a few of the simulations performed; see Fig. S27.

To verify that the superstructure obtained by our coarsegrained molecular dynamics model with respect to the input parameters, i.e., the results of Fig. 4, is the NCs' configuration with the lowest total free energy $\mathcal{F}$ [Eq. (4)], we estimate the total free energy variation $\Delta \mathcal{F}$ of a single $\mathrm{NC}$ that in the initial state is isolated at the interface and in the final state belongs to either a silicene-honeycomb or a square superstructure.
- In the square superstructure $(s)$, the $\mathrm{NC}$ forms four NC-NC bonds, gaining an energy $\simeq 4 \mathcal{E} / 2$ (the division by two is done since each bond is shared among two NCs). In addition, the NC pays an energy penalty to change its orientation at the interface [from $\{111\}$-up to $\{100\}$-up; see Fig. 3(a)], which is $u_{\varphi} \Delta \varphi^{2}+u_{\psi} \Delta \psi^{2}$ [see Eqs. (2) and (3)], where on average $\Delta \varphi$ $\simeq \Delta \psi \simeq 0.2 \pi$. Therefore, setting $u_{\psi}=u_{\varphi} / 2$, we have

$$
\Delta \mathcal{F}_{s} \simeq 2 \mathcal{E}+0.06 \pi^{2} u_{\varphi}
$$

- In the silicene-honeycomb superstructure $(h)$, the NC forms three $\mathrm{NC}-\mathrm{NC}$ bonds, gaining an energy $\simeq 3 \mathcal{E} / 2$. In addition, the NC pays an energy penalty to shift its height at the interface [see Fig. 3(a)], which is $u_{z}(\Delta z / \mathrm{nm})^{2}$ [see Eq. (1)], where on average $\Delta z \simeq 1.5 \mathrm{~nm}$. Therefore, 


$$
\Delta \mathcal{F}_{h} \simeq 1.5 \mathcal{E}+2.25 u_{z}
$$

With respect to the input parameters $\mathcal{E}, u_{\varphi}$, and $u_{z}$, if both $\Delta \mathcal{F}_{h}$ and $\Delta \mathcal{F}_{s}$ are greater than zero, then the NCs do not self-assemble. Otherwise, if $\Delta \mathcal{F}_{h}<\Delta \mathcal{F}_{s}$, then the NCs form silicene-honeycomb superstructures, while, if $\Delta \mathcal{F}_{s}<\Delta \mathcal{F}_{h}$, then the NCs form square superstructures. For example, in Fig. 5, we plot, for $\mathcal{E}=-1.0 \times 10^{-19}$ $\mathrm{J}, \Delta \mathcal{F}_{s}$ with respect to $u_{\varphi}$ and $\Delta \mathcal{F}_{h}$ for various values of $u_{z}$. For $u_{z}$ $=5 \times 10^{-21} \mathrm{~J}$, we see that $\Delta \mathcal{F}_{s}<\Delta \mathcal{F}_{h}$, i.e., the square lattice forms, for $u_{\varphi} \lesssim 1.0 \times 10^{-19} \mathrm{~J}$, and otherwise, $\Delta \mathcal{F}_{h}<\Delta \mathcal{F}_{s}$, i.e., the silicenehoneycomb superstructure forms, which is in line with the phase diagram of Fig. 4 for $\mathcal{E}=-1.0 \times 10^{-19} \mathrm{~J}$. If $u_{z}$ increases, the value of $u_{\varphi}$ above which square superstructures do not form increases, as predicted by Fig. 4. If $u_{z} \gtrsim 65 \times 10^{-19} \mathrm{~J}$, then $\Delta \mathcal{F}_{h}>0$, so silicenehoneycomb superstructures do not form for any value of $u_{\varphi}$, and square superstructures form only for $u_{\varphi} \lesssim 3 \times 10^{-19} \mathrm{~J}$, again in line with the predictions of Fig. 4 for $\mathcal{E}=-1.0 \times 10^{-19} \mathrm{~J}$. These estimations confirm that, in our simulations, the NCs evolve toward the configuration with the lowest total free energy $\mathcal{F}$ [Eq. (4)]. Of course, such a simple analytic model, based only on the NC's energetics in the various NCs' phases at the interface, neglects the NCs' entropy [i.e., the term $-T \cdot \mathcal{S}_{N}$ in $\mathcal{F}$; see Eq. (4)], while entropic effects are automatically included in the simulations with our coarse-grained molecular dynamics model (by the NCs' Brownian motion and by the NC-NC hard interactions). In addition, in Eqs. (9) and (10), we assume a homogeneous NCs' phase at the interface, so energetic contributions due to boundaries between different NCs' phases are neglected, while these effects are included in the simulations. Therefore, more precise results such as phase coexistence between different NCs' phases, disorder of NCs' superstructures, etc., can only be observed from the simulations with our coarse-grained molecular dynamics model. ${ }^{40}$ We also remark that our numerical model simulates the dynamics of the NCs at the interface, rather than simply extracting the lowest energy structure, thus allowing, for example, to find out if kinetically trapped NCs' configurations occur in certain regimes.

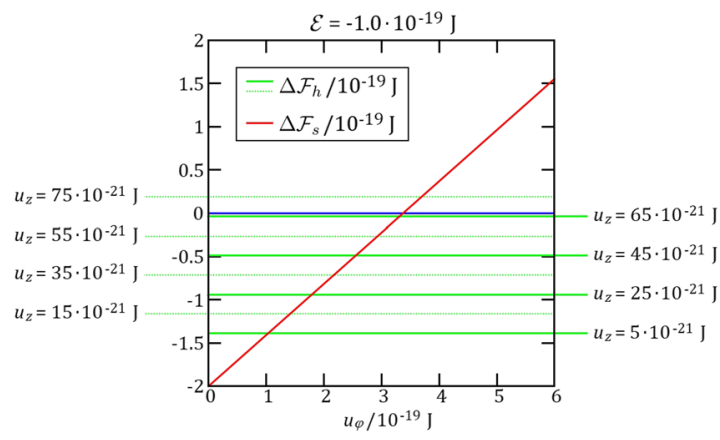

FIG. 5. Plot of $\Delta \mathcal{F}_{s}$ [Eq. (9)] with respect to $u_{\varphi}$ (red line) for $\mathcal{E}=-1.0 \times 10^{-19}$ $\mathrm{J}$ and of $\Delta \mathcal{F}_{h}$ [Eq. (10)] for $\mathcal{E}=-1.0 \times 10^{-19} \mathrm{~J}$ and various values of $u_{z}$ (green lines). If both $\Delta \mathcal{F}_{s}>0$ and $\Delta \mathcal{F}_{h}>0$, then the NCs do not self-assemble, otherwise, NCs form silicene-honeycomb superstructures for $\Delta \mathcal{F}_{h}<\Delta \mathcal{F}_{s}$ and NCs form square superstructures for $\Delta \mathcal{F}_{s}<\Delta \mathcal{F}_{h}$ (see the text).

\section{CALCULATIONS OF DIRECTIONAL ADSORPTION OF NCS AT THE DISPERSION-AIR INTERFACE}

In this section, we consider an isolated NC adsorbed at a flat fluid-fluid interface, say the NCs' dispersion-air interface, and calculate the NC's potential energy with respect to the NC's position and orientation at this interface and with respect to the NC's surface chemistry.

\section{A. Method}

We use a macroscopic model where the interface is treated as a possibly curved $2 \mathrm{D}$ surface, i.e., without thickness, and the two fluids forming the interface (i.e., the NCs' solvent and air) are treated as homogeneous. The NC is modeled as a polyhedral shape with a smooth surface. The energy of this fluid-fluid-NC system is ${ }^{40}$

$$
E=\gamma(S-A+W \cos \theta),
$$

where $S$ is the fluid-fluid interface area when the NC is adsorbed, $A$ is the fluid-fluid interface area when there is no NC, $W$ is the NC's surface area in contact with the fluid above the fluid-fluid interface (air), $\gamma$ is the fluid-fluid surface tension, and $\cos \theta$ is the cosine of Young's contact angle $\theta$ that is related to the surface tensions in the system by Young's law,

$$
\cos \theta=\frac{\gamma_{1}-\gamma_{2}}{\gamma}
$$

where $\gamma_{1}$ is the surface tension of the NC's surface with the fluid above the fluid-fluid interface (air) and $\gamma_{2}$ is the surface tension of the NC's surface with the fluid below the fluid-fluid interface (the solvent where the NC was initially dispersed). In this work, we consider several values of $\theta$, but always in the regime $\gamma_{1}-\gamma_{2} \geq 0$, which is the expected experimental condition since the NCs' surface, covered (partly or fully) by ligand molecules, has a stronger chemical affinity with the solvent than with air. Note that $E=0$ when the $\mathrm{NC}$ is desorbed from the solvent-air interface and immersed in the solvent bulk, so $E$ [Eq. (11)] is the energy bond of the NC to the interface. In the expression of the total free energy $\mathcal{F}$ of $N$ NCs at an interface [Eq. (4)], $E$ corresponds to $\mathcal{U}_{z}+\mathcal{U}_{\varphi}+\mathcal{U}_{\psi}$. That is, the external potentials $\mathcal{U}_{z}, \mathcal{U}_{\varphi}$, and $\mathcal{U}_{\psi}$ applied to the NCs in our coarse-grained molecular dynamics model (exploited for the results presented in Sec. II) are imposed to represent $E$ [Eq. (11)]. In Sec. III B, we predict $E$ for a single-adsorbed NC with respect to the NC's surface chemistry, using the model described here in Sec. III A.

Computing E [Eq. (11)] is, basically, a geometrical problem. While $A$ is just a constant defined by the problem, the surface areas $S$ and $W$ are variables that depend on the fluid-fluid interface equilibrium shape and on the $\mathrm{NC}$ 's position and orientation at the interface. Considering a flat fluid-fluid interface, the position and orientation of a NC can be univocally defined by three parameters. We use (see Fig. 2) the polar angle $\varphi$ of the NC's vertical axis, the internal Euler angle $\psi$ around the NC's vertical axis, and the $z$ coordinate of the NC center of mass, $z_{\mathcal{c}}$, where $z=0$ corresponds to the 
fluid-fluid interface plane. The so-called Pieranski approximation ${ }^{4}$ consists in assuming that the fluid-fluid interface shape remains flat when the NC adsorbs. In this approximation, the energy $E\left(z_{c}, \varphi, \psi\right)$ of the NC at the interface can be computed by calculating the intersection area $A-S\left(z_{c}, \varphi, \psi\right)$ between the NC's shape and the interface plane and the surface area $W\left(z_{c}, \varphi, \psi\right)$ of the NC cut above the fluid-fluid interface plane. Carrying out this calculation analytically is typically quite cumbersome, except for the most simple particle shapes, e.g., a sphere. Therefore, numerical techniques are usually used, e.g., a triangular tessellation technique ${ }^{21,22,46-50}$ or a hit-andmiss Monte Carlo method. ${ }^{51,52}$ However, when a micro- or nanoparticle is adsorbed at a fluid-fluid interface, capillary deformations can be induced in the equilibrium shape of the interface, affecting both $S\left(z_{c}, \varphi, \psi\right)$ and $W\left(z_{c}, \varphi, \psi\right)$. Although, at first glance, this might seem a negligible effect; it has been proven ${ }^{53}$ that neglecting capillary deformations can affect not just quantitatively but even qualitatively the energy $E\left(z_{\mathcal{c}}, \varphi, \psi\right)$ of a single-adsorbed particle, leading to false equilibrium orientations. In our numerical calculations to compute $E\left(z_{c}, \varphi, \psi\right)$, we include also the effects of the capillary deformations induced by the $\mathrm{NC}$ on the fluid-fluid interface equilibrium shape. For each NC's configuration $\left(z_{c}, \varphi, \psi\right)$ considered, we first use a simulated-annealing simulation (i.e., Monte Carlo) to equilibrate the fluid-fluid interface shape, i.e., to compute the interface shape that is the solution of the Young-Laplace equation with Young's law as a boundary condition on the three-phase contact line. ${ }^{54,55}$ During the simulated annealing, we place a vertical wall with the contact angle $\pi / 2$ around the NC-fluid-fluid system, to induce a flat fluidfluid interface far away from the NC. Once the equilibrium shape of the fluid-fluid interface is obtained from the simulated-annealing simulation, we use a tessellation technique approach to compute $E\left(z_{c}, \varphi, \psi\right)$.

The whole procedure to compute $E\left(z_{c}, \varphi, \psi\right)$ is sketched in Fig. 6; first, we define the position and orientation $\left(z_{c}, \varphi, \psi\right)$ of the NC at the interface, then we equilibrate the fluid-fluid interface shape, and finally, we compute $E$. By repeating this procedure for various $\left(z_{c}, \varphi, \psi\right)$, we obtain the energy landscape in $\left(z_{c}, \varphi, \psi\right)$. The simulated annealing to equilibrate the fluid-fluid interface shape can be performed either constraining the fluid volumes to remain constant or allowing the volume exchange between the fluids. In the latter case, the level of the fluid-fluid interface evolves toward its minimum- $E$ value, so the energy $E$ computed for the current NC orientation $(\varphi, \psi)$ is automatically minimized over the NC height $z_{c}$ at the interface. More details about our simulated-annealing procedure are illustrated in Refs. 54 and 55. Various applications of this numerical method for the equilibrium shape of fluid-fluid interfaces to micro- and nanoparticles at interfaces and to droplets in contact with complex substrates can be found in Refs. 40, 41, 53, and 56-61.

It is important to note that in our approach, we are neglecting diffuse-interface effects. If the considered NC is too small in size, the thickness of the fluid-fluid interface, which is zero in our model, affects the energy $E\left(z_{c}, \varphi, \psi\right)$. Comparing the results of Gupta et al. ${ }^{62}$ obtained by explicit-solvent molecular dynamics simulations with the results of Soligno et al. ${ }^{53}$ obtained by the sharp-interface model, we deduce that for the solvents of interest (e.g., toluene), the minimum NC's size to assume diffuse-interface effects negligible is around $3 \mathrm{~nm}$. Therefore, the results presented in Sec. III B are valid for NCs with a diameter larger than $\sim 3 \mathrm{~nm}$.

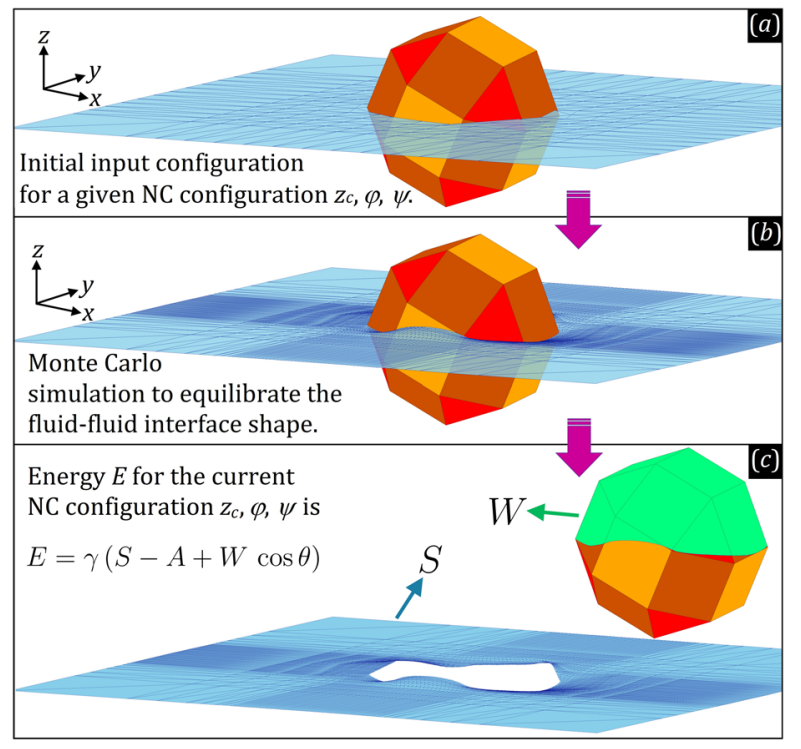

FIG. 6. Sketch illustrating our method to compute the energy landscape of a NC adsorbed at a fluid-fluid interface. For a given input configuration $\left(z_{c}, \varphi, \psi\right)$ of the $\mathrm{NC}$ at the interface (see Fig. 2), first we use a simulated-annealing approach to compute the equilibrium shape of the fluid-fluid interface [see (b)], starting from a flat interface [see (a)]. Then, the energy $E[E q$. (11)] is computed by calculating, with a triangular tessellation approach, the surface area of the fluid-fluid interface and the NC's surface area in contact with the fluid above the interface [see (c)]. By repeating this procedure for different NC's input configurations, the energy landscape $E\left(z_{c}, \varphi, \psi\right)$ is obtained.

Another approximation to keep in account while using our model is that we assume the NC's surface smooth, while in the experiments, the NC's surface is typically covered by ligand molecules (e.g., oleic acid) chemisorbed to it. However, while the head of the ligand molecules is attached to the NC's surface, we expect the tails of the ligand molecules to mix with the surrounding fluid molecules. ${ }^{63-65}$ Hence, we can assume that the ligand molecules chemisorbed on the NC's surface do not affect the NC's shape, but induce an "effective" surface tension of the NC's surface determined by the interactions of ligand-fluid molecules.

\section{B. Results}

Here, we present our results for the energy landscape $E\left(z_{c}, \varphi\right.$, $\psi)$ [Eq. (11)] of an isolated NC at a flat fluid-fluid interface (say, the NCs' dispersion-air interface), where (see Fig. 2) $\varphi$ is the polar angle of the NC's vertical axis, $\psi \in[0,2 \pi)$ is the NC's internal Euler angle around its vertical axis, and $z_{c}$ is the $z$ coordinate of the NC center of mass, with $z=0$ being the interface plane. We consider two different NC shapes: a rhombicuboctahedron [which is, in fact, a highly truncated cube; see Fig. 7(a)], typical for rocksalt PbSe NCs of 5-6 nm size, ${ }^{32}$ and a cantellated rhombicuboctahedron [which is a slightly truncated cube; see Fig. 7(b)], typical for larger rocksalt PbSe NCs. The key parameter (apart from the NC's shape) that dictates the interface-adsorption properties of the NC at the solventair interface is Young's contact angle $\theta$ [Eq. (12)]. Since we expect a 


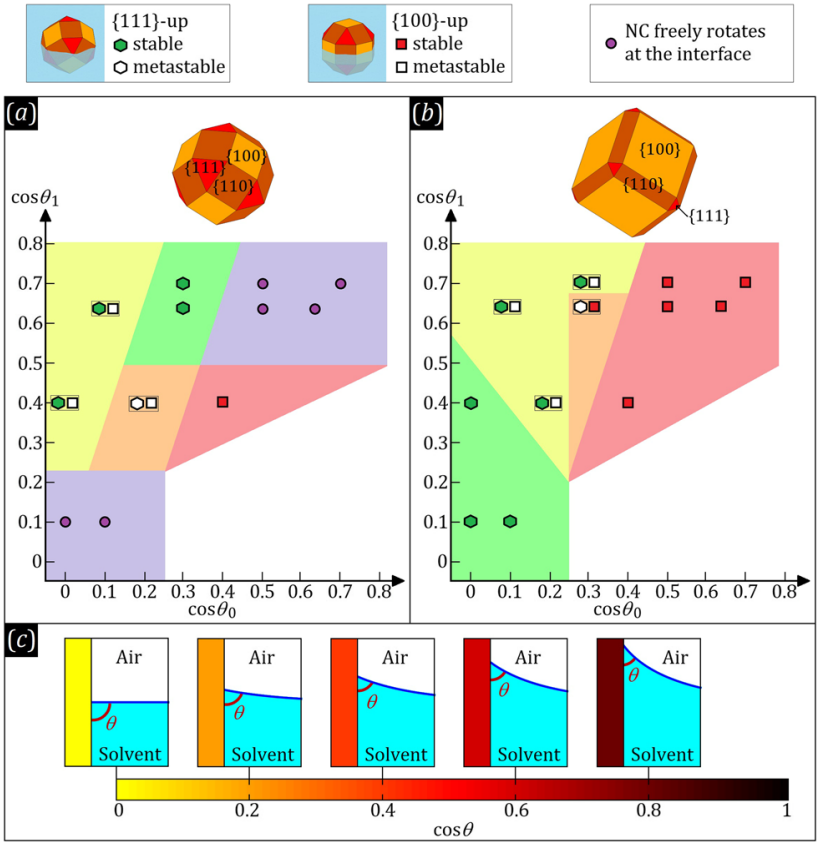

FIG. 7. Stable and metastable orientations predicted for an isolated NC, with (a) rhombicuboctahedron and (b) cantellated rhombicuboctahedron shapes, at the dispersion-air interface with respect to Young's contact angle $\theta_{0}$ of the NC's $\{100\}$ facets and Young's contact angle $\theta_{1}$ of the NC's $\{110\}$ and $\{111\}$ facets. The cantellation degree for the NC's shape in (b) is defined setting the side of a NC's $\{100\}$ facet to 0.75 times the distance between two opposite NC's $\{100\}$ facets. Where two stable or metastable orientations are grouped together, it means that they are found for the same combination of $\theta_{0}$ and $\theta_{1}$. The colored areas are a guide to the eye arbitrarily drawn to highlight the different situations predicted for the NC at the solvent-air interface: (green) only the $\{111\}$-up orientation is stable; (yellowgreen) the $\{111\}$-up orientation is stable, but the $\{100\}$-up orientation is metastable; (yellow-red) the $\{111\}$-up orientation is metastable; (red) the $\{100\}$-up orientation is stable; (violet) the $\mathrm{NC}$ has, essentially, orientation freedom at the interface. For the current experimental conditions for the synthesis of silicene-honeycomb superstructures, ${ }^{31,38}$ we estimate (see Ref. 40) that $\cos \theta_{1}=0.64$ and $\cos \theta_{0} \simeq 0.3$. In (c), we show the analytical shape of the solvent-air meniscus close to a vertical wall, for various values of $\cos \theta$, to help in visualizing the relation between $\cos \theta$ and $\theta$.

different density of ligand molecules covering the NC's $\{100\}$ facets compared to the NC's remaining facets, the effective surface tensions $\gamma_{1}, \gamma_{2}$ of the NC's $\{100\}$ facets with air and solvent, respectively, can be different from the effective surface tensions $\gamma_{1}, \gamma_{2}$ of the NC's remaining facets with air and solvent, respectively. That is, Young's contact angle $\theta$ [Eq. (12)] of the NC's $\{100\}$ facets, say $\theta_{0}$, can be different from Young's contact angle $\theta$ of the NC's remaining facets, say $\theta_{1}$. This effect can be included in our numerical method by rewriting Eq. (11) as

$$
E=\gamma\left(S-A+W_{0} \cos \theta_{0}+W_{1} \cos \theta_{1}\right),
$$

where $W_{0}$ is the total surface area of the NC's $\{100\}$ facets in contact with air and $W_{1}$ is the total surface area of the NC's $\{110\}$ and $\{111\}$ facets in contact with air (so, $W_{0}+W_{1}=W$, with $W$ the total surface area of the NC in contact with air). In the experiments for the synthesis of silicene-honeycomb superstructures, it is not clear which are the values of Young's contact angles $\theta_{0}$ and $\theta_{1}$, since these have never been measured (theoretically or experimentally), to the best of the authors' knowledge. In Ref. 40, we calculate $E\left(z_{c}, \varphi, \psi\right)$ for the PbSe NCs at the toluene-air interface setting $\cos \theta_{0}=0.30$ and $\cos \theta_{1}=0.64$. These values are an educated first guess obtained by assuming that the NC's facets covered by the oleic acid have the same surface tension of hexane. Here, we want to investigate how the energy landscape $E\left(z_{c}, \varphi, \psi\right)$ varies if we tune $\theta_{0}$ and $\theta_{1}$, to find out the most favorable conditions for the formation of silicenehoneycomb superstructures. Then, experimentally, it should be possible to direct the system toward these conditions by systematically regulating the various surface tensions, as we discuss in Sec. IV.

In Fig. 7(a), we report the rhombicuboctahedron NC's stable and metastable orientations at the solvent-air interface, obtained from $E(\varphi, \psi)$ minimized on $z_{c}$, as computed with the method described in Sec. III A, with respect to Young's contact angle $\theta_{0}$ of the NC's $\{100\}$ facets and with respect to Young's contact angle $\theta_{1}$ of the NC's $\{111\}$ and $\{110\}$ facets. As shown, the $\{111\}$-up orientation is found stable (a necessary condition to form silicenehoneycomb superstructures) for $0.1 \leq \cos \theta_{0} \leq 0.3$ and $\cos \theta_{1}$ $\geq 0.64$. A stable $\{111\}$-up orientation also arises for $\cos \theta_{0}=0$ and $\cos \theta_{1}=0.4$. In practice [see also Eq. (12)], the $\{111\}$-up orientation is stable for the rhombicuboctahedron NC when all the NC's facets have a higher chemical affinity with the solvent than with air, and in addition, the preference for the solvent is stronger on the NC's $\{111\}$ and $\{110\}$ facets, compared to the NC's $\{100\}$ facets. This is, actually, the condition in which, we believe, the experiments already are, since the ligand molecules chemisorbed on the NC's surface have a strong affinity with the solvent molecules, and ligand molecules are less dense on the NC's $\{100\}$ facets. Note that if the contact angle is the same on all the NC's facets, then the $\{111\}$ up orientation is never stable. So, the different surface chemistry of the NC's $\{100\}$ facets with respect to the NC's $\{111\}$ and $\{110\}$ facets is a necessary condition to stabilize the $\{111\}$-up orientation, at least for this NC's shape. These results are further discussed in Sec. IV.

In Fig. 7(b), we report the cantellated rhombicuboctahedron NC's stable and metastable orientations at the solvent-air interface, obtained from $E(\varphi, \psi)$ minimized on $z_{c}$, as computed with the method described in Sec. III A, with respect to Young's contact angle $\theta_{0}$ of the NC's $\{100\}$ facets and with respect to Young's contact angle $\theta_{1}$ of the NC's $\{111\}$ and $\{110\}$ facets. The results for this NC's shape show some important differences with respect to the NC's rhombicuboctahedron shape considered in Fig. 7(a). For $0 \leq \cos \theta_{0} \leq 1$ and $\cos \theta_{1}=0.1$, the NC adsorbs at the interface stably in the $\{111\}$-up orientation. These results are in line with the calculations presented in Refs. 53 and 59 for a NC with a similar shape and $\cos \theta_{0}=\cos \theta_{1}$ $=0$. For $\cos \theta_{1}=0.4$, the NC has a stable $\{111\}$-up orientation for $0 \leq \cos \theta_{0} \leq 0.2$. For $\cos \theta_{1}=0.64$, the $\mathrm{NC}$ has a stable $\{111\}$-up orientation for $\cos \theta_{0}=0.1$, but not for $\cos \theta_{0}>0.1$. For $\cos \theta_{1}=0.7$, the NC has a stable $\{111\}$-up orientation for $\cos \theta_{0}=0.3$, but not for $\cos \theta_{0}>0.3$. In practice [see also Eq. (12)], the $\{111\}$-up orientation is stable for the cantellated rhombicuboctahedron NC when the chemical affinity of all the NC's facets with air is similar to the chemical affinity of all the NC's facets with the solvent. If the chemical affinity of the NC's $\{111\}$ and $\{110\}$ facets with the solvent is 
increased with respect to their chemical affinity with air, the NC is also at equilibrium in the $\{111\}$-up orientation. However, if all the NC's facets have a stronger chemical affinity with the solvent than with air, then the NC prefers the $\{100\}$-up orientation. These results are further discussed in Sec. IV.

For the combinations of $\cos \theta_{0}$ and $\cos \theta_{1}$ that lead to a stable $\{111\}$-up interfacial orientation of the rhombicuboctahedron and cantellated rhombicuboctahedron NC, we report in Tables I and II, respectively, the values of $u_{\varphi}$ and $u_{\psi}$ obtained by fitting $E(\varphi, \psi)$, minimized on $z_{c}$, with $\mathcal{U}_{\varphi}(\varphi)$ [Eq. (2)] and $\mathcal{U}_{\psi}(\psi)$ [Eq. (3)], respectively, in a neighborhood of the minimum. We also report the value of $u_{z}$, obtained by fitting $E\left(z_{c}\right)$, computed for the NC in the $\{111\}$-up orientation, with $\mathcal{U}_{z}\left(z_{c}\right)$ [Eq. (1)], in a neighborhood of the minimum. As shown in Tables I and II, $u_{\psi}$ is always approximately equal to $u_{\varphi} / 2$, which is the condition used in the simulations with our coarsegrained molecular-dynamics model for the results shown in Fig. 4. The values of $u_{\varphi}$ and $u_{z}$, shown in Tables I and II (and obtained by the sharp-interface model illustrated in Sec. III A), can be compared with the assembled-phase diagrams in Fig. 4 (obtained by simulating with our coarse-grained molecular dynamics model, ${ }^{40}$ illustrated in Sec. I, the self-assembly of many NCs at the dispersion-air interface; see Sec. II) to find out whether the stable NC's $\{111\}$-up orientation, induced by a given combination of $\cos \theta_{0}$ and $\cos \theta_{1}$, leads to NCs' silicene-honeycomb or square self-assembly, or no self-assembly at all. This is further discussed in Sec. IV.

The plots of $E(\varphi, \psi)$, minimized on $z_{c}$, and of $E\left(z_{c}\right)$, computed for the NC in the $\{111\}$-up orientation, obtained with the method described in Sec. III A are shown in the supplementary material (see Figs. S1-S24) for the rhombicuboctahedron NC and for the cantellated rhombicuboctahedron $\mathrm{NC}$, for all values of $\theta_{0}$ and $\theta_{1}$ considered. In the cases where the NC's $\{111\}$-up orientation is found out to be stable (see Tables I and II), our fits of $E$ with $\mathcal{U}_{z}\left(z_{c}\right), \mathcal{U}_{\varphi}(\varphi)$, and $\mathcal{U}_{\psi}(\psi)$ are also shown. In the supplementary material (see Figs. S25 and S26), we also show a 3D view of the $\mathrm{NC}$ and of the solventair interface equilibrium shape close to the $\mathrm{NC}$, as computed with the method of Sec. III A, for all the NC's stable and metastable orientations reported in Figs. 7(a) and 7(b).

TABLE I. Summary of the stable $\{111\}$-up orientations of a rhombicuboctahedron NC at the solvent-air interface that we found from our calculations; see Fig. 7. Young's contact angle is $\theta_{0}$ on the NC's $\{100\}$ facets and $\theta_{1}$ on the NC's $\{110\}$ and $\{111\}$ facets. For each stable $\{111\}$-up orientation, i.e., at equilibrium, we report the energy $E\left[E q\right.$. (13)] and the values of the parameters $u_{z}, u_{\varphi}$, and $u_{\psi}$ obtained by fitting the landscape $E\left(z_{c}, \varphi, \psi\right)$ with $\mathcal{U}_{z}\left(z_{c}\right)$ [Eq. (1)], $\mathcal{U}_{\varphi}(\varphi)$ [Eq. (2)], and $\mathcal{U}_{\psi}(\psi)$ [Eq. (3)], respectively, in a neighborhood of the minimum, where $\left(z_{c}, \varphi, \psi\right)$ is the NC's configuration at the interface (see Fig. 2), $L$ is the NC's size (precisely, the distance between two opposite NC's $\{100\}$ facets), and $\gamma$ is the solvent-air surface tension. For the typical experimental values $\gamma=0.028 \mathrm{~N} / \mathrm{m}$ and $L=6 \mathrm{~nm}$, it follows $\gamma L^{2} \simeq 10.0 \times 10^{-19}$ $\mathrm{J}$ and $\gamma \mathrm{nm}^{2}=0.28 \times 10^{-19} \mathrm{~J}$.

\begin{tabular}{lccccc}
\hline \hline $\cos \theta_{0}$ & $\cos \theta_{1}$ & $E /\left(\gamma L^{2}\right)$ & $u_{\varphi} /\left(\gamma L^{2}\right)$ & $u_{\psi} /\left(\gamma L^{2}\right)$ & $u_{z} /\left(\gamma \mathrm{nm}^{2}\right)$ \\
\hline 0.30 & 0.70 & -0.198 & 0.322 & 0.159 & 1.283 \\
0.30 & 0.64 & -0.232 & 0.293 & 0.195 & 1.408 \\
0.10 & 0.64 & -0.325 & 0.380 & 0.231 & 1.796 \\
0.20 & 0.40 & -0.420 & 0.184 & 0.098 & 1.650 \\
0 & 0.40 & -0.522 & 0.227 & 0.176 & 1.850 \\
\hline \hline
\end{tabular}

TABLE II. Summary of the stable $\{111\}$-up orientations of a cantellated rhombicuboctahedron NC at the solvent-air interface that we found from our calculations; see Fig. 7. Young's contact angle is $\theta_{0}$ on the NC's $\{100\}$ facets and $\theta_{1}$ on the NC's $\{110\}$ and $\{111\}$ facets. For each stable $\{111\}$-up orientation, i.e., at equilibrium, we report the energy $E$ [Eq. (13)] and the values of the parameters $u_{z}, u_{\varphi}$, and $u_{\psi}$ obtained by fitting the landscape $E\left(z_{c}, \varphi, \psi\right)$ with $\mathcal{U}_{z}\left(z_{c}\right)$ [Eq. (1)], $\mathcal{U}_{\varphi}(\varphi)$ [Eq. (2)], and $\mathcal{U}_{\psi}(\psi)$ [Eq. (3)], respectively, in a neighborhood of the minimum, where $\left(z_{c}, \varphi, \psi\right)$ is the NC's configuration at the interface (see Fig. 2), $L$ is the NC's size (precisely, the distance between two opposite NC's $\{100\}$ facets), and $\gamma$ is the solvent-air surface tension. For the typical experimental values $\gamma=0.028 \mathrm{~N} / \mathrm{m}$ and $L=6 \mathrm{~nm}$, it follows $\gamma L^{2} \simeq 10.0$ $\times 10^{-19} \mathrm{~J}$ and $\gamma \mathrm{nm}^{2}=0.28 \times 10^{-19} \mathrm{~J}$.

\begin{tabular}{cccccc}
\hline \hline $\cos \theta_{0}$ & $\cos \theta_{1}$ & $E /\left(\gamma L^{2}\right)$ & $u_{\varphi} /\left(\gamma L^{2}\right)$ & $u_{\psi} /\left(\gamma L^{2}\right)$ & $u_{z} /\left(\gamma \mathrm{nm}^{2}\right)$ \\
\hline 0.30 & 0.70 & -0.407 & 0.298 & 0.226 & 1.445 \\
0.30 & 0.64 & -0.432 & 0.346 & 0.171 & 1.458 \\
0.10 & 0.64 & -0.738 & 0.539 & 0.323 & 1.957 \\
0.20 & 0.40 & -0.695 & 0.337 & 0.196 & 1.662 \\
0.00 & 0.40 & -1.02 & 0.604 & 0.291 & 1.947 \\
0.10 & 0.10 & -1.031 & 0.376 & 0.169 & 1.623 \\
0.00 & 0.10 & -1.201 & 0.384 & 0.197 & 1.759 \\
\hline \hline
\end{tabular}

\section{DISCUSSION}

First, we discuss the conditions predicted in Sec. III B from the sharp-interface numerical model for the stability of the NC's $\{111\}$ up orientation at the NCs' dispersion-air interface (see Fig. 7) that is necessary for forming NCs' silicene-honeycomb superstructures at this interface.

- For the NC with a rhombicuboctahedron shape [i.e., a highly truncated cube, typically of rocksalt $\mathrm{PbSe}$ with the size 5-6 nm; see Fig. 7(a)], the surface chemistry of the NC's $\{111\}$ and $\{110\}$ facets needs to be different from the surface chemistry of the NC's $\{100\}$ facets. In addition,

- The NC's $\{111\}$ and $\{110\}$ facets strongly prefer the solvent to air (the contact angle $\theta_{1}$ for these facets is such that $\left.\cos \theta_{1} \geq 0.64\right)$, and the NC's $\{100\}$ facets also prefer the solvent to air, but less strongly (the contact angle $\theta_{0}$ for these facets is such that $0 \leq \cos \theta_{0} \leq 0.3$ ).

- Alternatively, the NC's $\{111\}$ and $\{110\}$ facets prefer the solvent to air, but less strongly $\left(\cos \theta_{1}=0.4\right)$, and the NC's $\{100\}$ facets have equal affinity with solvent and $\operatorname{air}\left(\cos \theta_{0}=0\right)$.

The current experiments for synthesizing silicene-honeycomb superstructures are expected to fall within this range of conditions. In Ref. 40, we estimated $\cos \theta_{1}=0.64$ and $\cos \theta_{0}=0.3$, assuming that the NC's facets fully covered by the oleic acid have the same surface tension of hexane. In Fig. 7(a), these values of $\cos \theta_{0}$ and $\cos \theta_{1}$ fall within the area where the $\{111\}$-up orientation is stable, and such an area is very narrowly extended around these values. This means that, for such a NC's shape, the experiments already are very close to the conditions that allow silicene-honeycomb superstructures. As a matter of fact, silicene-honeycomb superstructures are observed, as a confirmation that our predictions are realistic; the only small region in the $\left(\theta_{0}, \theta_{1}\right)$ plane where we predict the $\{111\}$-up 
orientation stable is where the experiments are expected to fall. Therefore, to improve the current synthesis of silicenehoneycomb superstructures using such a NC's shape, it is unwise to drastically change the experimental conditions. Instead, one should focus, we believe, on a systematic and mild tuning of the various surface tensions in the system to slightly move around the current experimental conditions, probing where silicene-honeycomb formation is most favored.

- For the NC with a cantellated rhombicuboctahedron shape [i.e., a slightly truncated cube, typically of rocksalt $\mathrm{PbSe}$ with a size larger than $6 \mathrm{~nm}$; see Fig. 7(b)], the conditions to induce a stable $\{111\}$-up orientation are different. For the current experimental conditions, expected to be around the values $\cos \theta_{1}=0.64$ and $\cos \theta_{0}=0.3$, a NC with such a shape adsorbs at the interface with a $\{100\}$ facet pointing upward [see Fig. 7(b)]; hence, it forms square superstructures. Therefore, when larger and more cubic-like PbSe NCs are used, for the current experimental conditions, we expect square superstructures to become more dominant. To induce a stable $\{111\}$-up orientation with such a NC's shape, the following conditions are necessary:

- The NC's $\{111\}$ and $\{110\}$ facets have any contact angle $\theta_{1}$ among those considered $\left(0 \leq \cos \theta_{1} \leq 0.7\right)$. That is, these facets can have a much stronger chemical affinity with the solvent than with air, or they can also have the same chemical affinity with solvent and with air.

- The NC's $\{100\}$ facets have any contact angle $\theta_{0}$ such that $0 \leq \cos \theta_{0} \leq 0.2$ (if $\cos \theta_{1}=0.7$, then the condition for $\theta_{0}$ is more relaxed, i.e., $\left.0 \leq \cos \theta_{0} \leq 0.3\right)$, that is, the NC's $\{100\}$ facets have a similar chemical affinity with air and with the solvent, rather than a strong preference for the solvent.

In summary, we predict that more cubic-like NCs (i.e., with the cantellated rhombicuboctahedron shape) offer a much wider range of surface chemistry conditions (compared to the rhombicuboctahedron NCs) to induce a stable $\{111\}$-up orientation. Therefore, using more cubic-like NCs seems a viable strategy to improve the synthesis of silicenehoneycomb superstructures. However, the current experimental conditions are expected to fall outside the regime where the more cubic-like NCs have a stable $\{111\}$-up orientation. So, some changes in the current experimental procedures are required. A viable strategy to tune the current experimental conditions toward the regime where the more cubic-like NCs are, at the dispersion-air interface, stable in the $\{111\}$-up orientation (see Fig. 7) consists, in our opinion, in lowering as much as possible $\left|\cos \theta_{1}\right|$ and $\left|\cos \theta_{0}\right|$, which can be achieved simply by using a (much) higher solvent-air surface tension; see Eq. (12). In addition, $\left|\cos \theta_{1}\right|$ and $\left|\cos \theta_{0}\right|$ can also be lowered by regulating the interactions of the NC's surface with air and solvent such that the NC's surface has an as-much-similar-as-possible chemical affinity with the solvent and with air (this can be achieved, e.g., by using different ligand molecules chemisorbed on the NC's surface or by changing the molecular composition of either the solvent or air).

Second, we discuss, on the basis of the results predicted in Sec. II, when a stable $\{111\}$-up orientation of the NC at the dispersion-air interface leads to the formation of silicenehoneycomb superstructures. As shown in Fig. 7 (see Sec. III), only certain combinations of the NC's shape and surface chemistry allow the stability of the NC's $\{111\}$-up orientation at the interface. As illustrated in Secs. I and II, the stability of the NC's $\{111\}$-up orientation at the interface is a necessary condition for the formation of silicene-honeycomb superstructures, but it does not guarantee it. For a given NC-NC attachment energy $\mathcal{E}$, only certain ranges of $u_{z}$ and $u_{\varphi}$ allow the formation of silicene-honeycomb superstructures (see Fig. 4). We remind (see Sec. I) that $u_{z}$ is the parameter regulating the forces keeping the NCs in-plane at the interface [see Eq. (1)] and $u_{\varphi}$ is the parameter regulating the forces orienting the NCs with a $\{111\}$ facet upward at the interface [see Eq. (2)]. If $u_{z}$ is too high, the NCs are forced to stay in-plane at the interface, so they cannot form silicene-honeycomb superstructures [NC-NC attachment by opposite $\{100\}$ facets cannot occur; see Fig. 3(a)]. If $u_{\varphi}$ is too low, the NCs lose their $\{111\}$-up orientation at the interface while remaining in-plane [to attach by opposite $\{100\}$ facets by forming linear and square superstructures; see Fig. 3(a)], so in this case, the NCs do not form silicene-honeycomb superstructures. Note that the predictions for the stability of the NC's $\{111\}$-up orientation at the interface (see Sec. III) are NC-size independent and surface-tension independent, since the predicted shape of the energy landscape $E\left(z_{c}, \varphi, \psi\right)$ [Eqs. (11) and (13)], with $\left(z_{c}, \varphi, \psi\right)$ the NC's configuration at the interface (see Fig. 2), depends on the NC's shape and Young's contact angle, but not on $L$ (NC's size) and $\gamma$ (solventair surface tension), which are only scaling factors for $E\left(z_{c}, \varphi, \psi\right)$. Indeed, in Tables I and II, we report the values of $u_{\varphi}$, fitted from $E(\varphi, \psi)$, in units of $\gamma L^{2}$, with $\gamma$ the solvent-air surface tension and $L$ the NC's size (precisely, $L$ is the distance between two opposite $\{100\}$ facets of the NC), and the values of $u_{z}$, fitted from $E\left(z_{c}\right)$, in units of $\gamma \mathrm{nm}^{2}$. Setting $\gamma=0.028 \mathrm{~N} / \mathrm{m}$, that is, the toluene-air surface tension, and $L=6 \mathrm{~nm}$, that is, the typical experimental NC's size (and the NC's size used in the self-assembly simulations of Sec. II), it follows $\gamma L^{2} \simeq 10.0 \times 10^{-19} \mathrm{~J}$ and $\gamma \mathrm{nm}^{2}=0.28 \times 10^{-19} \mathrm{~J}$. Using these values for $\gamma$ and $L$, consequently, $u_{\varphi}$ ranges from $1.8 \times 10^{-19} \mathrm{~J}\left(\cos \theta_{0}=0.2, \cos \theta_{1}=0.4\right.$, rhombicuboctahedron NC) until $6.0 \times 10^{-19} \mathrm{~J}\left(\cos \theta_{0}=0, \cos \theta_{1}=0.4\right.$, cantellated rhombicuboctahedron NC) and $u_{z}$ ranges from $0.36 \times 10^{-19} \mathrm{~J}\left(\cos \theta_{0}=0.3\right.$, $\cos \theta_{1}=0.7$, rhombicuboctahedron NC) until $0.55 \times 10^{-19} \mathrm{~J}\left(\cos \theta_{0}\right.$ $=0.1, \cos \theta_{1}=0.64$, cantellated rhombicuboctahedron NC). Inspecting the assembled-phase diagrams in Fig. 4, we see that, within these ranges of $u_{\varphi}$ and $u_{z}$, we are mostly in the coexistence region (i.e., mixed $\sim$ green, $\sim$ red, and $\sim$ blue boxes), corresponding to the formation of silicene-honeycomb superstructures in coexistence with square superstructures and with nonbond NCs. For the lowest value of $\mathcal{E}$ considered (i.e., $0.7 \times 10^{-19} \mathrm{~J}$ ), more $\sim$ blue boxes occur within these ranges of $u_{\varphi}$ and $u_{z}$, i.e., the non-bonded-NC phase is dominant. For the highest value of $\mathcal{E}$ considered (i.e., $1.3 \times 10^{-19} \mathrm{~J}$ ), more $\sim$ red boxes occur within these ranges of $u_{\varphi}$ and $u_{z}$, i.e., the square superstructure phase is dominant. For the mid value of $\mathcal{E}$ considered (i.e., $1.0 \times 10^{-19} \mathrm{~J}$ ), we are, within these ranges of $u_{\varphi}$ and $u_{z}$, closer to the area where $\sim$ green boxes are, i.e., where formation 
of silicene-honeycomb superstructures dominates. For example, to induce a self-assembly of rhombicuboctahedron NCs in silicenehoneycomb superstructures, for $\gamma=0.028 \mathrm{~N} / \mathrm{m}$ and $L=6 \mathrm{~nm}$, we see that $\cos \theta_{0}=0.30$ and $\cos \theta_{1}=0.70$ is a good combination. Indeed, for these values of $\theta_{0}$ and $\theta_{1}$, we have $u_{\varphi}=3.2 \times 10^{-19} \mathrm{~J}$ and $u_{z}=0.36 \times 10^{-19} \mathrm{~J}$, leading to the coexistence region, i.e., where also silicene-honeycomb superstructures form, in the assembledphase diagrams of Fig. 4, for most of the values of $\mathcal{E}$ considered. Using, instead of $\cos \theta_{0}=0.20, \cos \theta_{1}=0.40$ does not promote the self-assembly of rhombicuboctahedron NCs in silicene-honeycomb superstructures although the values of $\theta_{0}$ and $\theta_{1}$ induce a stable $\{111\}$-up orientation for an isolated rhombicuboctahedron NC at the dispersion-air interface. Indeed, for $\cos \theta_{0}=0.20$ and $\cos \theta_{1}$ $=0.40$, we have $u_{\varphi}=1.8 \times 10^{-19} \mathrm{~J}$ and $u_{z}=0.46 \times 10^{-19} \mathrm{~J}$, leading to the region where only square superstructures form, i.e., where only $\sim$ red boxes are, in the assembled-phase diagrams of Fig. 4, for most of the values of $\mathcal{E}$ considered. So, since the combination $\cos \theta_{0}=0.20, \cos \theta_{1}=0.40$ leads to only a square superlattice formation while the combination $\cos \theta_{0}=0.30, \cos \theta_{1}=0.70$ also leads to a silicene-honeycomb superstructure formation, we deduce that, to promote the self-assembly of rhombicuboctahedron NCs in silicene-honeycomb superstructures, the higher $\cos \theta_{1}$ is with respect to $\cos \theta_{0}$, the better. We remark that if the fluid-fluid surface tension $\gamma$ is changed from the value $0.028 \mathrm{~N} / \mathrm{m}$ considered here (for example, in the first part of the discussion, we suggest that using a higher fluid-fluid surface tension can help induce a stable $\{111\}$ up interfacial orientation for the cantellated rhombicuboctahedron NC), then the predicted values of $u_{\varphi}$ and $u_{z}$, in Tables I and II, are rescaled according to the new $\gamma$, and from these values, the accessible regions in the assembled-phase diagrams of Fig. 4 are determined. Finally, we point out that, using $\gamma=0.028 \mathrm{~N} / \mathrm{m}$, the bond energy $E$ [Eqs. (11) and (13)] of the NC at the interface at equilibrium ( $E$ $=0$ means the $\mathrm{NC}$ is desorbed in the solvent) ranges (see Tables I and II) from $-2.0 \times 10^{-19} \mathrm{~J} \simeq 49 k_{B} T_{r}$ until $-5.2 \times 10^{-19} \mathrm{~J} \simeq 127$ $k_{B} T_{r}$ for the rhombicuboctahedron NC and from $-4.1 \times 10^{-19} \mathrm{~J}$ $\simeq 100 k_{B} T_{r}$ until $-12.0 \times 10^{-19} \mathrm{~J} \simeq 292 k_{B} T_{r}$ for the cantellated rhombicuboctahedron NC. We remark that these values correspond to the energy cost for completely desorbing the $\mathrm{NC}$ from the dispersion-air interface such that the $\mathrm{NC}$ is fully immersed in the solvent. However, the partial desorption of a NC from the interface, occurring when NCs form silicene-honeycomb superstructures, requires a much lower energy cost ( $\sim 5$ to $10 k_{B} T_{r}$; see Fig. 1) and is compensated by the energy gain of NCs attaching by opposite $\{100\}$ facets.

A complementary strategy to favor NCs' self-assembly in silicene-honeycomb superstructures could rely on using NCs with a magnetic moment (with the same direction in each $\mathrm{NC}$ and with an intensity small enough to not affect NC-NC interactions at the interface). By applying an opportune magnetic field to the system, the NCs' orientation at the interface could be regulated to stabilize the $\{111\}$-up orientation. In addition, the parameter $u_{\varphi}$ could be directly tuned without affecting the parameter $u_{z}$. By disentangling $u_{\varphi}$ from $u_{z}$, the system could be driven into regions of the phase diagram of Fig. 4 not allowed by solely the NCs' chemistry at the interface (i.e., by solely the NC's Young's contact angle, which leads to the predictions for $u_{z}, u_{\varphi}$, and $u_{\psi}$ presented in Sec. III). In this way, the regimes where NCs only form silicene-honeycomb superstructures could more easily be reached.

\section{CONCLUSIONS AND OUTLOOK}

In conclusion, in this work, we theoretically investigate the conditions that induce an interfacial self-assembly of PbSe NCs in silicene-honeycomb or square superstructures. In Sec. II, we use a coarse-grained molecular dynamics model to simulate the selfassembly of 6-nm-sized NCs at the solvent-air interface with respect to the input parameters regulating the various forces involved in the self-assembly. As a result, we draw extensive diagrams of the NCs' assembled phase with respect to these parameters (see Fig. 4), showing which are the regimes that induce the formation of silicenehoneycomb superstructures and which are the regimes that induce the formation of square superstructures. Then, in Sec. III, we use a sharp-interface numerical model to predict the stability of the NC's $\{111\}$-up orientation (necessary condition for the interfacial selfassembly in silicene-honeycomb superstructures) for PbSe NCs at various fluid-fluid interfaces, showing that only certain combinations of the NC's surface chemistry and shape allow a stable $\{111\}$-up orientation (see Fig. 7). Finally, in Sec. IV, we combine the results of Secs. II and III to speculate some experimental guidelines to attempt a better control over the PbSe silicene-honeycomb superlattice formation. In future interdisciplinary theory-experiment works, we plan to test these guidelines.

In addition, there are still many aspects in the experimental synthesis of PbSe silicene-honeycomb superlattices that need to be clarified. We plan to investigate them in the future, both theoretically and experimentally, with the final goal of reaching a better control over NCs' interfacial self-assembly. Some of the aspects that we plan to investigate are the following:

- How and when, during the experiments, the ligand molecules chemisorbed on the NCs' $\{100\}$ facets start desorbing from these facets.

- A more precise estimation, for the experiments, of key parameters such as $\mathcal{E}$ (NC-NC attachment energy) and Young's contact angle of interface-adsorbed NCs.

- The interface-adsorption energy $E$ [Eq. (11)] for aggregates of two or more NCs, after they performed the oriented attachment. (In Sec. III, we predicted the energy landscape $E$ for a single NC. When two NCs merge together becoming a single block, the two-NC block energy landscape $E$ is not necessarily the sum of the two single-NC energy landscapes, although in some cases this might be a good approximation. Understanding how the energy landscape $E$ evolves for NC aggregates might reveal useful insights into how to favor the formation of silicene-honeycomb superstructures.)

- Other NC shapes, compounds, or ligand molecules that might also lead to honeycomb or silicene-honeycomb interfacial self-assembly.

\section{SUPPLEMENTARY MATERIAL}

See the supplementary material for an extended version of the assembled-phase diagrams of Fig. 4 (reporting the numerical values of the order parameters for all the 6272 simulations performed in Sec. II), for a snapshot of the final NCs' configuration for some illustrative simulations among the 6272 simulations performed in Sec. II, for the plots of the energy landscape $E\left(z_{c}, \varphi, \psi\right)$ for all $\theta_{0}$ and $\theta_{1}$ combinations considered for the rhombicuboctahedron and cantellated 
rhombicuboctahedron NCs in Sec. III B, and for a 3D view of the $\mathrm{NC}$ and of the solvent-air interface equilibrium shape close to the $\mathrm{NC}$ for the various stable and metastable NC's orientations found in Sec. III B and reported in Fig. 7.

\section{ACKNOWLEDGMENTS}

The authors acknowledge financial support by the Dutch NWO Physics with the FOM Program No. 152 "Engineering Dirac physics in semiconductor superlattices," the Dutch NWO Chemistry with the TOPPUNT No. 718.015.002 "Superficial superstructures: control over colloidal ordering at interfaces," and the ERC Advanced Grant No. 692691 "FIRST STEP."

\section{REFERENCES}

${ }^{1}$ M. A. Boles, M. Engel, and D. V. Talapin, "Self-assembly of colloidal nanocrystals: From intricate structures to functional materials," Chem. Rev. 116(18), 11220-11289 (2016)

${ }^{2}$ A. C. Berends and C. de Mello Donega, "Ultrathin one- and two-dimensional colloidal semiconductor nanocrystals: Pushing quantum confinement to the limit," J. Phys. Chem. Lett. 8(17), 4077-4090 (2017).

${ }^{3}$ F. Reincke, S. G. Hickey, W. K. Kegel, and D. Vanmaekelbergh, "Spontaneous assembly of a monolayer of charged gold nanocrystals at the water/oil interface," Angew. Chem., Int. Ed. 43(4), 458-462 (2004).

${ }^{4}$ H. Duan, D. Wang, D. G. Kurth, and H. Möhwald, "Directing self-assembly of nanoparticles at water/oil interfaces," Angew. Chem., Int. Ed. 43(42), 5639-5642 (2004).

${ }^{5}$ K. M. Gattás-Asfura, C. A. Constantine, M. J. Lynn, D. A. Thimann, X. Ji, and R. M. Leblanc, "Characterization and 2D self-assembly of CdSe quantum dots at the air-water interface," J. Am. Chem. Soc. 127(42), 14640-14646 (2005).

${ }^{6}$ Y.-J. Li, W.-J. Huang, and S.-G. Sun, "A universal approach for the self-assembly of hydrophilic nanoparticles into ordered monolayer films at a toluene/water interface," Angew. Chem., Int. Ed. 45(16), 2537-2539 (2006).

${ }^{7}$ J. Wang, D. Wang, N. S. Sobal, M. Giersig, M. Jiang, and H. Möhwald, "Stepwise directing of nanocrystals to self-assemble at water/oil interfaces," Angew. Chem., Int. Ed. 45(47), 7963-7966 (2006).

${ }^{8}$ T. P. Bigioni, X.-M. Lin, T. T. Nguyen, E. I. Corwin, T. A. Witten, and H. M. Jaeger, "Kinetically driven self assembly of highly ordered nanoparticle monolayers," Nat. Mater. 5(4), 265-270 (2006).

${ }^{9}$ A. Böker, J. He, T. Emrick, and T. P. Russell, "Self-assembly of nanoparticles at interfaces," Soft Matter 3, 1231-1248 (2007).

${ }^{10}$ Y. Shi, C. Tu, Q. Zhu, H. Qian, J. Ren, C. Liu, X. Zhu, D. Yan, E. S.-W. Kong, and P. He, "Self-assembly of CdTe nanocrystals at the water/oil interface by amphiphilic hyperbranched polymers," Nanotechnology 19, 445609 (2008).

${ }^{11}$ A. Dong, J. Chen, P. M. Vora, J. M. Kikkawa, and C. B. Murray, "Binary nanocrystal superlattice membranes self-assembled at the liquid-air interface," Nature 466, 474 (2010).

${ }^{12}$ M. D. Goodman, L. Zhao, K. A. DeRocher, J. Wang, S. K. Mallapragada, and Z. Lin, "Self-assembly of CdTe tetrapods into network monolayers at the air/water interface," ACS Nano 4(4), 2043-2050 (2010)

${ }^{13}$ Z. Jiang, X.-M. Lin, M. Sprung, S. Narayanan, and J. Wang, "Capturing the crystalline phase of two-dimensional nanocrystal superlattices in action," Nano Lett. 10(3), 799-803 (2010).

${ }^{14}$ L. Cheng, A. Liu, S. Peng, and H. Duan, "Responsive plasmonic assemblies of amphiphilic nanocrystals at oil-water interfaces," ACS Nano 4(10), 6098-6104 (2010).

${ }^{15}$ J. Chen, A. Dong, J. Cai, X. Ye, Y. Kang, J. M. Kikkawa, and C. B. Murray, "Collective dipolar interactions in self-assembled magnetic binary nanocrystal superlattice membranes," Nano Lett. 10(12), 5103-5108 (2010).

${ }^{16}$ Y. Justo, I. Moreels, K. Lambert, and Z. Hens, "Langmuir-Blodgett monolayers of colloidal lead chalcogenide quantum dots: Morphology and photoluminescence," Nanotechnology 21, 295606 (2010).
${ }^{17}$ D. Vanmaekelbergh, "Self-assembly of colloidal nanocrystals as route to novel classes of nanostructured materials," Nano Today 6(4), 419-437 (2011).

${ }^{18} \mathrm{H}$. Ma and J. Hao, "Ordered patterns and structures via interfacial self-assembly: Superlattices, honeycomb structures and coffee rings," Chem. Soc. Rev. 40, 54575471 (2011).

${ }^{19}$ J. J. Choi, K. Bian, W. J. Baumgardner, D.-M. Smilgies, and T. Hanrath, "Interface-induced nucleation, orientational alignment and symmetry transformations in nanocube superlattices," Nano Lett. 12(9), 4791-4798 (2012).

${ }^{20}$ F. Pietra, F. T. Rabouw, W. H. Evers, D. V. Byelov, A. V. Petukhov, C. de Mello Donegá, and D. Vanmaekelbergh, "Semiconductor nanorod self-assembly at the liquid/air interface studied by in situ GISAXS and ex situ TEM," Nano Lett. 12(11), 5515-5523 (2012).

${ }^{21}$ H. Evers, B. Goris, S. Bals, J. Casavola, M. de Graaf, R. van Roij, M. Dijkstra, and D. Vanmaekelbergh, "Low-dimensional semiconductor superlattices formed by geometric control over nanocrystal attachment," Nano Lett. 13, 2317 (2013).

${ }^{22}$ W. van der Stam, A. Gantapara, Q. A. Akkerman, G. Soligno, J. M. Meeldijk, R. van Roij, M. Dijkstra, and C. de Mello Donega, "Self-assembly of colloidal hexagonal bipyramid and bifrustum-shaped $\mathrm{ZnS}$ nanocrystals into two dimensional superstructures," Nano Lett. 14, 1032 (2014).

${ }^{23}$ C. S. S. Sandeep, J. M. Azpiroz, W. H. Evers, S. C. Boehme, I. Moreels, S. Kinge, L. D. A. Siebbeles, I. Infante, and A. J. Houtepen, "Epitaxially connected PbSe quantum-dot films: Controlled neck formation and optoelectronic properties," ACS Nano 8(11), 11499-11511 (2014).

${ }^{24}$ W. Walravens, J. De Roo, E. Drijvers, S. ten Brinck, E. Solano, J. Dendooven, C. Detavernier, I. Infante, and Z. Hens, "Chemically triggered formation of twodimensional epitaxial quantum dot superlattices," ACS Nano 10(7), 6861-6870 (2016).

${ }^{25}$ B. H. Savitzky, R. Hovden, K. Whitham, J. Yang, F. Wise, T. Hanrath, and L. F. Kourkoutis, "Propagation of structural disorder in epitaxially connected quantum dot solids from atomic to micron scale," Nano Lett. 16(9), 5714-5718 (2016).

${ }^{26}$ J. J. Geuchies, C. van Overbeek, E. H., B. Goris, A. de Backer, A. P. Gantapara, F. T. Rabouw, J. Hilhorst, J. L. Peters, O. Konovalov, A. V. Petukhov, M. Dijkstra, L. D. A. Siebbeles, S. van Aert, S. Bals, and D. Vanmaekelbergh, "In situ study of the formation mechanism of two-dimensional superlattices from PbSe nanocrystals," Nat. Mater. 15, 1248 (2016).

${ }^{27}$ S. Maiti, A. André, R. Banerjee, J. Hagenlocher, O. Konovalov, F. Schreiber, and $\mathrm{M}$. Scheele, "Monitoring self-assembly and ligand exchange of PbS nanocrystal superlattices at the liquid/air interface in real time," J. Phys. Chem. Lett. 9(4), 739744 (2018).

${ }^{28}$ H. Itasaka, K.-I. Mimura, and K. Kato, "Extra surfactant-assisted self-assembly of highly ordered monolayers of $\mathrm{BaTiO}_{3}$ nanocubes at the air-water interface," Nanomaterials 8, 739 (2018).

${ }^{29}$ A. Castelli, J. de Graaf, S. Marras, R. Brescia, L. Goldoni, L. Manna, and M. P. Arciniegas, "Understanding and tailoring ligand interactions in the selfassembly of branched colloidal nanocrystals into planar superlattices," Nat. Commun. 9(1), 1141 (2018).

${ }^{30}$ C. van Overbeek, J. L. Peters, S. A. P. van Rossum, M. Smits, M. A. van Huis, and D. Vanmaekelbergh, "Interfacial self-assembly and oriented attachment in the family of $\mathrm{PbX}(\mathrm{X}=\mathrm{S}, \mathrm{Se}, \mathrm{Te})$ nanocrystals," J. Phys. Chem. C 122(23), 1246412473 (2018).

${ }^{31}$ M. P. Boneschanscher, W. H. Evers, J. J. Geuchies, T. Altantzis, B. Goris, F. T. Rabouw, S. A. P. van Rossum, H. S. J. van der Zant, L. D. A. Siebbeles, G. Van Tendeloo, I. Swart, J. Hilhorst, A. V. Petukhov, S. Bals, and D. Vanmaekelbergh, "Long-range orientation and atomic attachment of nanocrystals in $2 \mathrm{D}$ honeycomb superlattices," Science 344, 1377 (2014).

${ }^{32}$ J. L. Peters, K. H. W. van den Bos, S. Van Aert, B. Goris, S. Bals, and D. Vanmaekelbergh, "Ligand-induced shape transformation of PbSe nanocrystals," Chem. Mater. 29(9), 4122-4128 (2017).

${ }^{33}$ R. K. Mallavajula and L. A. Archer, "Nanocrystal self-assembly assisted by oriented attachment," Angew. Chem., Int. Ed. 50(3), 578-580 (2011).

${ }^{34}$ J. J. De Yoreo, P. U. P. A. Gilbert, N. A. J. M. Sommerdijk, R. L. Penn, S. Whitelam, D. Joester, H. Zhang, J. D. Rimer, A. Navrotsky, J. F. Banfield, A. F. Wallace, F. M. Michel, F. C. Meldrum, H. Cölfen, and P. M. Dove, "Crystallization 
by particle attachment in synthetic, biogenic, and geologic environments," Science 349(6247), aaa6760 (2015).

${ }^{35}$ E. Kalesaki, C. Delerue, C. Morais Smith, W. Beugeling, G. Allan, and D. Vanmaekelbergh, "Dirac cones, topological edge states, and nontrivial flat bands in two-dimensional semiconductors with a honeycomb nanogeometry," Phys. Rev. X 4, 011010 (2014).

${ }^{36}$ C. Delerue and D. Vanmaekelbergh, "Electronic band structure of zinc blende $\mathrm{CdSe}$ and rock salt $\mathrm{PbSe}$ semiconductors with silicene-type honeycomb geometry," 2D Mater. 2(3), 034008 (2015).

${ }^{37}$ W. Beugeling, E. Kalesaki, C. Delerue, Y. M. Niquet, D. Vanmaekelbergh, and C. Morais Smith, "Topological states in multi-orbital HgTe honeycomb lattices," Nat. Commun. 6, 1 (2015).

${ }^{38}$ J. L. Peters, T. Altantzis, I. Lobato, M. A. Jazi, C. van Overbeek, S. Bals, D. Vanmaekelbergh, and S. B. Sinai, "Mono- and multilayer silicene-type honeycomb lattices by oriented attachment of PbSe nanocrystals: Synthesis, structural characterization, and analysis of the disorder," Chem. Mater. 30(14), 4831-4837 (2018).

${ }^{39} \mathrm{~K}$. Whitham, D.-M. Smilgies, and T. Hanrath, "Entropic, enthalpic, and kinetic aspects of interfacial nanocrystal superlattice assembly and attachment," Chem. Mater. 30(1), 54-63 (2018).

${ }^{40} \mathrm{G}$. Soligno and D. Vanmaekelbergh, "Understanding the formation of PbSe honeycomb superstructures by dynamics simulations," Phys. Rev. X 9, 021015 (2019).

${ }^{41}$ J. J. Geuchies, G. Soligno, E. Geraffy, C. P. Hendrikx, C. van Overbeek, F. Montanarella, M. R. Slot, O. V. Konovalov, A. V. Petukhov, and D. Vanmaekelbergh, "Unravelling three-dimensional adsorption geometry of PbSe nanocrystal monolayers at a liquid-air interface using synchrotron $\mathrm{x}$-ray scattering techniques" (submitted).

${ }^{42}$ M. R. Khadilkar and F. A. Escobedo, "Phase behavior of polyhedral nanoparticles in parallel plate confinement," Soft Matter 12, 1506-1516 (2016).

${ }^{43} \mathrm{Z}$. Fan and M. Grünwald, "Orientational order in self-assembled nanocrystal superlattices,” J. Am. Chem. Soc. 141(5), 1980-1988 (2019).

${ }^{44} \mathrm{H}$. Zhang and J. F. Banfield, "Energy calculations predict nanoparticle attachment orientations and asymmetric crystal formation," J. Phys. Chem. Lett. 3(19), 2882-2886 (2012)

${ }^{45}$ P. Pieranski, “Two-dimensional interfacial colloidal crystals," Phys. Rev. Lett. 45, 569-572 (1980).

${ }^{46}$ J. de Graaf, M. Dijkstra, and R. van Roij, “A triangular tessellation scheme for the adsorption free energy at the liquid-liquid interface: Towards non-convex patterned colloids," Phys. Rev. E 80, 051405 (2009).

${ }^{47}$ J. de Graaf, M. Dijkstra, and R. van Roij, "Adsorption trajectories and freeenergy separatrices for colloidal particles in contact with a liquid-liquid interface," J. Chem. Phys. 132, 164902 (2010).

${ }^{48}$ A. R. Morgan, N. Ballard, L. A. Rochford, G. Nurumbetov, T. S. Skelhon, and S. A. F. Bon, "Understanding the multiple orientations of isolated superellipsoidal hematite particles at the oil-water interface," Soft Matter 9, 487 (2013).

${ }^{49}$ B. Peng, G. Soligno, M. Kamp, B. de Nijs, J. de Graaf, M. Dijkstra, R. van Roij, A. van Blaaderen, and A. Imhof, "Site-specific growth of polymer on silica rods," Soft Matter 10, 9644 (2014).
${ }^{50} \mathrm{~N}$. Ballard and S. A. F. Bon, "Equilibrium orientations of non-spherical and chemically anisotropic particles at liquid-liquid interfaces and the effect on emulsion stability,” J. Colloid Interface Sci. 448, 533 (2015).

${ }^{51}$ B. J. Park and D. Lee, "Configuration of nonspherical amphiphilic particles at a fluid-fluid interface," Soft Matter 8, 7690 (2012).

${ }^{52}$ B. J. Park and D. Lee, "Equilibrium orientation of nonspherical Janus particles at fluid-fluid interfaces," ACS Nano 6, 782 (2012).

${ }^{53}$ G. Soligno, M. Dijkstra, and R. van Roij, "Self-assembly of cubes into 2D hexagonal and honeycomb lattices by hexapolar capillary interactions," Phys. Rev. Lett. 116, 258001 (2016).

${ }^{54}$ G. Soligno, M. Dijkstra, and R. van Roij, "The equilibrium shape of fluid-fluid interfaces: Derivation and a new numerical method for Young's and YoungLaplace equations," J. Chem. Phys. 141, 244702 (2014).

${ }^{55} \mathrm{G}$. Soligno, "Droplets, capillary interactions, and self-assembly from the equilibrium shape of fluid-fluid interfaces," Ph.D. thesis, Utrecht University, 2017, ISBN: 978-94-6332-124-2.

${ }^{56}$ M. Vis, J. Opdam, I. S. J. van 't Oor, G. Soligno, R. van Roij, R. H. Tromp, and B. H. Erné, "Water-in-water emulsions stabilized by nanoplates," ACS Macro Lett. 4, 965 (2015).

${ }^{57}$ I. Dević, G. Soligno, M. Dijkstra, R. van Roij, X. Zhang, and D. Lohse, "Sessile nanodroplets on elliptical patches of enhanced lyophilicity," Langmuir 33(11), 2744-2749 (2017).

${ }^{58}$ M. Kamp, G. Soligno, F. Hagemans, B. Peng, A. Imhof, R. van Roij, and A. van Blaaderen, "Regiospecific nucleation and growth of silane coupling agent droplets onto colloidal particles," J. Phys. Chem. C 121(36), 19989-19998 (2017).

${ }^{59}$ G. Soligno, M. Dijkstra, and R. van Roij, "Self-assembly of cubic colloidal particles at fluid-fluid interfaces by hexapolar capillary interactions," Soft Matter 14, 42-60 (2018)

${ }^{60}$ C. Anzivino, F. Chang, G. Soligno, R. van Roij, W. K. Kegel, and M. Dijkstra, "Equilibrium configurations and capillary interactions of Janus dumbbells and spherocylinders at fluid-fluid interfaces," Soft Matter 15, 2638-2647 (2019).

${ }^{61}$ M. Zanini, A. Cingolani, C.-P. Hsu, M. A. Fernandez Rodriguez, G. Soligno, A. Beltzung, S. Caimi, D. M. Mitrano, G. Storti, and L. Isa, "Mechanical phase inversion of pickering emulsions via metastable wetting of rough colloids," Soft Matter 15, 7888 (2019).

${ }^{62}$ U. Gupta, T. Hanrath, and F. A. Escobedo, "Modeling the orientational and positional behavior of polyhedral nanoparticles at fluid-fluid interfaces," Phys. Rev. Mater. 1, 055602 (2017).

${ }^{63}$ R. J. K. Udayana Ranatunga, R. J. B. Kalescky, C.-c. Chiu, and S. O. Nielsen, "Molecular dynamics simulations of surfactant functionalized nanoparticles in the vicinity of an oil/water interface," J. Phys. Chem. C 114(28), 12151-12157 (2010).

${ }^{64}$ K. Schwenke, L. Isa, D. L. Cheung, and E. Del Gado, "Conformations and effective interactions of polymer-coated nanoparticles at liquid interfaces," Langmuir 30(42), 12578-12586 (2014).

${ }^{65}$ A. P. Kaushik and P. Clancy, "Explicit all-atom modeling of realistically sized ligand-capped nanocrystals,” J. Chem. Phys. 136(11), 114702 (2012). 